\title{
INTEGRABILITY OF UNITARY REPRESENTATIONS ON REPRODUCING KERNEL SPACES
}

\author{
STÉPHANE MERIGON, KARL-HERMANN NEEB, AND GESTUR ÓLAFSSON
}

\begin{abstract}
Let $\mathfrak{g}$ be a Banach-Lie algebra and $\tau: \mathfrak{g} \rightarrow \mathfrak{g}$ an involution. Write $\mathfrak{g}=\mathfrak{h} \oplus \mathfrak{q}$ for the eigenspace decomposition of $\mathfrak{g}$ with respect to $\tau$ and $\mathfrak{g}^{c}:=\mathfrak{h} \oplus i \mathfrak{q}$ for the dual Lie algebra. In this article we show the integrability of two types of infinitesimally unitary representations of $\mathfrak{g}^{c}$. The first class of representation is determined by a smooth positive definite kernel $K$ on a locally convex manifold $M$. The kernel is assumed to satisfy a natural invariance condition with respect to an infinitesimal action $\beta: \mathfrak{g} \rightarrow \mathcal{V}(M)$ by locally integrable vector fields that is compatible with a smooth action of a connected Lie group $H$ with Lie algebra $\mathfrak{h}$. The second class is constructed from a positive definite kernel corresponding to a positive definite distribution $K \in C^{-\infty}(M \times M)$ on a finite dimensional smooth manifold $M$ which satisfies a similar invariance condition with respect to a homomorphism $\beta: \mathfrak{g} \rightarrow \mathcal{V}(M)$. As a consequence, we get a generalization of the Lüscher-Mack Theorem which applies to a class of semigroups that need not have a polar decomposition. Our integrability results also apply naturally to local representations and representations arising in the context of reflection positivity.
\end{abstract}

\section{INTRODUCTION}

Let $G$ be a Banach-Lie group, let $\tau$ be an involutive automorphism of $G$ and let $H$ be an open subgroup of $G^{\tau}$. We call the triple $(G, H, \tau)$, respectively the pair $(G, \tau)$, a symmetric Lie group. We use the same notation for the involution induced on the Lie algebra $\mathfrak{g}$ of $G$ and call $(\mathfrak{g}, \mathfrak{h}, \tau)$, respectively $(\mathfrak{g}, \tau)$, a symmetric Lie algebra. Write $\mathfrak{g}=\mathfrak{h} \oplus \mathfrak{q}=\operatorname{ker}(\tau-\mathbf{1}) \oplus \operatorname{ker}(\tau+\mathbf{1})$ for the eigenspace decomposition of $\mathfrak{g}$ with respect to $\tau$. We have $[\mathfrak{h}, \mathfrak{h}] \subseteq \mathfrak{h},[\mathfrak{q}, \mathfrak{q}] \subseteq \mathfrak{h}$ and $[\mathfrak{h}, \mathfrak{q}] \subset \mathfrak{q}$. Thus $\mathfrak{g}^{c}:=\mathfrak{h} \oplus i \mathfrak{q}$ is a Lie algebra and $\tau^{c}(x+i y):=x-i y$ leads to the symmetric Lie algebra $\left(\mathfrak{g}^{c}, \tau^{c}\right)$, called the $c$-dual of $(\mathfrak{g}, \tau)$. Note that the $c$-dual of $\left(\mathfrak{g}^{c}, \tau^{c}\right)$ is the original symmetric Lie algebra $(\mathfrak{g}, \tau)$. We denote by $G^{c}$ a (connected) simply connected Lie group with Lie algebra $\mathfrak{g}^{c}$ and note that it is unique up to isomorphism.

When $\mathfrak{g}$ is a semisimple Lie algebra and $\tau$ is a Cartan involution, then $c$-duality corresponds to the well-known duality between Riemannian symmetric spaces of

Received by the editors June 10, 2014 and, in revised form, June 11, 2014 July 11, 2014 and October 1, 2014.

2010 Mathematics Subject Classification. 17B15, 22E30, 22E70.

Key words and phrases. Unitary representations, finite and infinite dimensional Lie groups, manifolds, reproducing kernel.

The first author would like to thank Louisiana State University for their hospitality during his visit in 2014 when most of the work on this article was done.

The research of the second author was supported by DFG-grant NE 413/7-2, SPP "Representation Theory".

The research of the third author was supported by NSF grant DMS-1101337, "Representation Theory and Harmonic Analysis on Homogeneous Spaces". 
non-compact type and compact type already studied by É. Cartan almost a century ago.

In this paper we address the following integrability problem: Suppose we are given a unitary representation $\left(\pi_{H}, \mathcal{H}\right)$ of $H$, a subspace $\mathcal{D} \subseteq \mathcal{H}$, and a representation $\beta_{c}: \mathfrak{g}^{c} \rightarrow \operatorname{End}(\mathcal{D})$ by skew-symmetric operators on $\mathcal{D}$ such that $\beta_{c}(x)=\left.\mathrm{d} \pi_{H}(x)\right|_{\mathcal{D}}$ for $x \in \mathfrak{h}$. When is there a unitary representation $\pi^{c}$ of $G^{c}$ on $\mathcal{H}$ with $\mathcal{D} \subseteq \mathcal{H}^{\infty}$ and $\left.\mathrm{d} \pi^{c}(x)\right|_{\mathcal{D}}=\beta_{c}(x)$ for $x \in \mathfrak{g}^{c}$ ? In this sense we are asking for integrability criteria for a representation of the pair $\left(\mathfrak{g}^{c}, H\right)$ to a representation $\pi^{c}$ of the group $G^{c}$. If the operators $i \beta_{c}(x), x \in \mathfrak{q}$, are essentially selfadjoint, then $\pi^{c}$ is uniquely determined.

Note that, for the one-dimensional Lie algebra $\mathfrak{g}=\mathbb{R}, \tau=-\mathrm{id}$, and $H=\{\mathbf{1}\}$, the problem is equivalent to finding selfadjoint extensions of a symmetric operator. In the general case a crucial step in the passage from $\left(\mathfrak{g}^{c}, H\right)$ to $G^{c}$ is the existence of selfadjoint extensions of the operators $i \beta_{c}(y)$ for $y \in i \mathfrak{q}$, so that Stone's Theorem leads to corresponding unitary one-parameter groups. To achieve this step we shall use Fröhlich's criterion for essential selfadjointness of a symmetric operator $A$ which derives this proprty from the existence of sufficiently many solutions of the ODE $\dot{\gamma}(t)=A \gamma(t)$ ([Fro80]). In our context this is much more natural than to use Nelson's Criterion ([Nel59, Lemma 5.1]) which is based on the existence of analytic vectors. One can actually translate between these two perspectives because any analytic vector provides a convergent power series solving the ODE and, conversely, the solutions of the ODE are actually analytic on open intervals (cf. [Sh84]).

Our main motivation to study this kind of integrability problem comes from our recent work on reflection positive unitary representations of $(G, \tau)$ by the last two authors (cf. [NO13, NO14]). Originally, reflection positivity (also called Osterwalder-Schrader positivity) was part of a duality between euclidean quantum field theories and relativistic quantum field theories ([OS73]). It can be realized by analytic continuation in the time variable from the real to the imaginary axis. A central difficulty in this approach is to show that the infinitesimal relativistic system obtained form the euclidean one can be integrated to the relativistic symmetry group, that is, that the corresponding representation of the Lie algebra of the Poincaré group can be integrated to a unitary representation of the group. The duality between the Poincaré group and the euclidean motion group is a special case of the $c$-duality introduced above.

Another very interesting occurrence of $c$-duality in representation theory is obtained as follows. Assume that $(G, H, \tau)$ is a simple symmetric Lie group and that $H$ is connected. Let $\theta$ be a Cartan involution commuting with $\tau$. We further assume that the center of $G^{\theta \tau}$ is one dimensional. Then $G^{c}$ is a hermitian Lie group, $\theta^{c}=\theta \tau$ is a Cartan involution on $G^{c}$ and $D:=G^{c} / K^{c}, K^{c}=\left(G^{c}\right)^{\theta^{c}}$, is a bounded symmetric domain. Furthermore, $\tau$ defines an anti-holomorphic involution on $D$ with fixed point set $D^{\tau} \cong H /\left(H \cap K^{c}\right)$. The compact dual $K /(K \cap H)$ of $D^{\tau}$ turns out to be an $R$-symmetric space (and all $R$-symmetric spaces are obtained this way). Its conformal group is locally isomorphic to $G$. In this case $c$-duality should lead, on the level of representations, to a correspondence between the maximally degenerate principal series of $G$ and the unitary highest weight representations of $G^{c}$. This has been discussed in JO198, JO100 for the case where $D^{c}$ is a tube type domain and in [NO14, Sch86] for related special cases. When one considers infinite dimensional versions of the preceding groups, $c$-duality also plays a crucial role in the study of the unitary representations of $H$ ( $\mathrm{Ol} 84, \mathrm{O} 190, \mathrm{Ne13}, \mathrm{MN14})$. 
In the literature one finds essentially three types of representations that lead by analytic continuation to a unitary representation of the simply connected Lie group $G^{c}$ with Lie algebra $\mathfrak{g}^{c}$ :

(L) local representations of $G$ for which the adjoint operation corresponds to $g \mapsto g^{\sharp}:=\tau(g)^{-1}$ and the representation is global on $H$ (cf. [Jo86, Jo87]),

(RP) reflection positive representations of $G$ ([JO198, JO100, NO13, Pr92]),

(LM) involutive representation of a subsemigroup $S \subseteq G$ with polar decomposition $S=H \exp W$, where $W \neq \emptyset$ is an $\operatorname{Ad}(H)$-invariant open convex cone in $\mathfrak{q}$ (the Lüscher-Mack Theorem [LM75, MN12]).

Each of these types leads more or less easily to a representation $\left(\beta_{c}, \pi_{H}\right)$ of $\left(\mathfrak{g}^{c}, H\right)$ as above.

In this paper we develop a uniform approach to the integrability of such pairs $\left(\beta_{c}, \pi_{H}\right)$ that is based on reproducing kernel techniques. It leads to much simpler proofs, applies to Banach-Lie groups, and for type (LM) gives rise to a version of the Lüscher-Mack Theorem that applies to semigroups without a polar decomposition and even to representations $\left(\beta_{c}, \pi_{H}\right)$ realized in reproducing kernel spaces on open $H$-right invariant domains in $G$ which are not even semigroups, and this is crucial for the applications to type (RP) (cf. Theorem 5.18 and Example 5.14). For type (L) it also allows us to avoid some technical conditions that were used in the definition of local representations in Jo86, Jo87.

The key idea is to realize the representations $\left(\beta_{c}, \pi_{H}\right)$ in a geometric setting which is rich enough to imply integrability to a representation of $G^{c}$. This is achieved by considering Hilbert spaces $\mathcal{H}$ defined by a smooth reproducing kernel $K$ on a locally convex manifold $M \mathbb{1}$ and which are compatible with a smooth action $(\beta, \sigma)$ of $(\mathfrak{g}, H)$, which means that $\sigma: M \times H \rightarrow M$ is a smooth right action and $\beta: \mathfrak{g} \rightarrow \mathcal{V}(M)$ a homomorphism of Lie algebras for which the map $\widehat{\beta}: \mathfrak{g} \times M \rightarrow$ $T M,(x, m) \mapsto \beta(x)(m)$ is smooth, $\dot{\sigma}(x)=\beta(x)$ for $x \in \mathfrak{h}$, where $\dot{\sigma}$ denotes the derived action, and each vector field $\beta(x), x \in \mathfrak{q}$, is locally integrable, i.e., generates a local flow. In this context the compatibility between $K$ and $(\beta, \sigma)$ can be expressed by

$$
\mathcal{L}_{\beta(x)}^{1} K=-\mathcal{L}_{\beta(\tau x)}^{2} K \quad \text { for } \quad x \in \mathfrak{g} .
$$

Here $\mathcal{L}^{1}$ and $\mathcal{L}^{2}$ denote the Lie derivatives with respect to the first and second variable, respectively. Using a geometric version of Fröhlich's Theorem on selfadjoint operators and corresponding local linear semiflows [Fro80, see Theorems 4.5] and 4.6 in this article, we show that this action leads to a representation of $\mathfrak{g}_{\mathbb{C}}$ on a dense domain $\mathcal{D}$ in the corresponding reproducing kernel Hilbert space $\mathcal{H}_{K} \subseteq C^{\infty}(M)$ for which $\mathfrak{g}^{c}$ acts by essentially skew-adjoint operators. The proof that this representation integrates to a unitary representation of $G^{c}$ then relies on the results in Mer11.

If $M$ is a finite dimensional manifold, a similar but much more general situation arises when $K$ is a positive definite distribution on $M \times M$. It is remarkable that in this case one can prove integrability along the same lines as for the case when $K$ is a smooth kernel. Here typical examples are obtained from so-called reflection positive representations of $(G, S, \tau)$, where $S \subseteq G$ is a $\sharp$-invariant open subsemigroup. If $\nu$

\footnotetext{
${ }^{1}$ This means a smooth manifold modeled on a locally convex space; see $\mathrm{Ne} 06$ for details.
} 
is a reflection positive distribution vector, then

$$
K_{\nu}(f, g)=\left\langle\pi^{-\infty}\left(g * f^{*}\right) \nu, \nu\right\rangle \quad \text { for } \quad f, g \in C_{c}^{\infty}(G)
$$

defines a positive definite distribution on $S \times S$ to which our results apply (cf. NO14]).

The article is organized as follows. To keep our setting as flexible as possible, we introduce in Section 2 the concept of a locally integrable vector field on a locally convex manifold. By definition, a vector field is locally integrable if it is the velocity field of a local flow and the main point of Section 2 is to show that any such vector field determines a unique maximal local flow. For Banach manifolds, every smooth vector field is locally integrable, but this is not the case for Fréchet spaces.

In Section 3, Lie derivatives of locally integrable vector fields are studied in more detail. The most important result here is Lemma 3.4, asserting the compatibility of the adjoint representation of $\mathfrak{g}$ and the transformation of the corresponding vector fields under flows. It will be used in a crucial way in the proof of the integrability theorems.

In Section 4 we use Fröhlich's Theorem to show that a vector field which is symmetric with respect to a smooth positive definite kernel $K$ on $M$ gives rise, by its Lie derivative, to a selfadjoint operator on $\mathcal{H}_{K}$.

Section 5 is the central section of this article. Here we prove the integrability of the representation $\left(\beta_{c}, \pi_{H}\right)$ on the reproducing kernel space $\mathcal{H}_{K}$ if (1.1) is satisfied (Theorem 5.12). As a rather easy consequence, we obtain the following generalization of the Lüscher-Mack Theorem: If $S=S H \subseteq G$ is a $\sharp$-invariant subsemigroup with non-empty interior, then any smooth $*$-representation of $S$ "extends analytically" to a uniquely determined unitary representation of $G^{c}$.

In Section [ 6 we first recall the definition of a local representation of a symmetric Lie group $(G, H, \tau)$ from Jo86, Jo87 and show in two different situations how it leads to a unitary representation of $G^{c}$. In the first case, where $G$ is finite dimensional, we give a simplified proof of Jorgensen's integrability to $G^{c}$. In the second case $G$ can be a Banach-Lie group, we assume that the local representation is defined on an open 1-neighborhood $U$ with $U=U H$, and that the corresponding action map $U \times \mathcal{D} \rightarrow \mathcal{H}$ is smooth for some topology on $\mathcal{D}$ for which the inclusion $\mathcal{D} \rightarrow \mathcal{H}$ is continuous. Then integrability can be derived from Theorem 5.12

In Section 7 we assume that the manifold $M$ is finite dimensional, so that the concept of a distribution on $M$ is defined. Here our main result is a generalization of Theorem 5.12 to the situation where the kernel $K$ is a distribution on $M \times M$ satisfying (1.1) in a suitable sense (Theorem 7.9). Again, the key tool is a suitable version of Fröhlich's Theorem in this context. This result applies immediately to reflection positive distributions, for which it leads to an important class of reflection positive representations of $(G, \tau)$, for which a corresponding unitary representation $\pi^{c}$ of $G^{c}$ exists.

Throughout this article $C^{-\infty}(M)$ denotes the space of distributions on a manifold $M$ and is defined as the space of continuous conjugate linear functionals on the space $C_{c}^{\infty}(M)$ of complex-valued smooth compactly supported functions. By $T(M)$ we denote the tangent bundle of $M$ and if $\varphi: M \rightarrow N$ is a smooth map between two manifolds, then $T(\varphi)=T \varphi: T(M) \rightarrow T(N)$ is the corresponding tangent map. If a group $G$ acts from the left, respectively right, on the manifold $M$, then the action is denoted by $(g, x) \mapsto g . x$, respectively $x . g$. If $V$ is a topological vector, then $V^{\sharp}$ stands for the space of continuous conjugate linear functionals on $V$. For 
a Hilbert space $\mathcal{H}$ we denote by $\mathrm{U}(\mathcal{H})$ the group of unitary operators on $\mathcal{H}$ and by $B(\mathcal{H})$ the space of bounded operators on $\mathcal{H}$. If $\pi$ is a unitary representation on $\mathcal{H}$ then $\mathcal{H}^{\infty}$ denotes the space of smooth vectors, endowed with its natural Fréchet topology (cf. [Ne10]), and $\mathcal{H}^{-\infty}=\left(\mathcal{H}^{\infty}\right)^{\sharp}$.

\section{LOCAL FLOWS ON LOCALLY CONVEX MANIFOLDS}

A smooth manifold $M$ modeled on a locally convex space is called a locally convex manifold. Throughout this section, all manifolds are assumed to be locally convex. We denote by $\mathcal{V}(M)$ the Lie algebra of smooth vector fields on $M$ (cf. [Ne06]).

In this section, we show that the well-known correspondence between smooth local flows and vector fields can be generalized to locally convex manifolds, the main difference being that not every vector field generates a local flow.

For a subset $\mathcal{D} \subset \mathbb{R} \times M, m \in M$ and $t \in \mathbb{R}$ let

$$
I_{m}:=\{s \in \mathbb{R}:(s, m) \in \mathcal{D}\} \quad \text { and } \quad M_{t}:=\{m \in M:(t, m) \in \mathcal{D}\} .
$$

Definition 2.1. Let $M$ be a smooth manifold. A local flow on $M$ is a smooth map $\Phi: \mathcal{D} \rightarrow M,(t, x) \mapsto \Phi_{t}(x)$, where $\mathcal{D} \subseteq \mathbb{R} \times M$ is an open subset containing $\{0\} \times M$, such that for each $x \in M$ the set $I_{x}$ is an interval containing 0 , and

$$
\Phi_{0}=\operatorname{id}_{M} \quad \text { and } \quad \Phi_{t} \Phi_{s}(x)=\Phi_{s+t}(x)
$$

hold for all $t, s, x$ for which both sides are defined. The maps

$$
\gamma_{x}: I_{x} \rightarrow M, \quad t \mapsto \Phi_{t}(x)
$$

are called the flow lines. The flow $\Phi$ is said to be global if $\mathcal{D}=\mathbb{R} \times M$.

Remark 2.2. Let $\Phi: \mathcal{D} \rightarrow M$ be a local flow. For each $t \in \mathbb{R}$, the subset $M_{t} \subseteq M$ is open, the map

$$
\Phi_{t}: M_{t} \rightarrow M, \quad m \mapsto \Phi(t, m)
$$

is smooth and, for $\Phi_{t}(m) \in M_{-t}$, we have $\Phi_{-t}\left(\Phi_{t}(m)\right)=m$.

If $\Phi: \mathcal{D} \rightarrow M$ is a local flow, then its velocity field

$$
X^{\Phi}(x):=\left.\frac{d}{d t}\right|_{t=0} \Phi_{t}(x)=\alpha_{x}^{\prime}(0)
$$

is a smooth vector field on $M$. From (2.1) we immediately obtain for $t \in \mathbb{R}$

$$
\left.T\left(\Phi_{t}\right) \circ X^{\Phi}\right|_{M_{t}}=X^{\Phi} \circ \Phi_{t} .
$$

Definition 2.3. Let $M$ be a smooth manifold.

(a) A smooth vector field $X \in \mathcal{V}(M)$ is called locally integrable if it is the velocity field of some smooth local flow.

(b) Let $I \subseteq \mathbb{R}$ be an open interval containing 0. A differentiable map $\gamma: I \rightarrow M$ is called an integral curve of $X$ if

$$
\gamma^{\prime}(t)=X(\gamma(t)) \quad \text { for each } \quad t \in I .
$$

(c) If $J \supseteq I$ is an interval containing $I$, then an integral curve $\eta: J \rightarrow M$ is called an extension of $\gamma$ if $\left.\eta\right|_{I}=\gamma$. An integral curve $\gamma$ is said to be maximal if it has no proper extension.

Note that (2.3) implies that the curve $\gamma^{\prime}$ in $T M$ is continuous and further that, if $\gamma$ is $C^{k}$, then $\gamma^{\prime}$ is also $C^{k}$. Therefore integral curves are automatically smooth. 
Example 2.4 (cf. [Ne06, Example II.3.11], [Ham82, 5.6.1]). We give examples of linear ODEs on Fréchet spaces for which multiple solutions to initial value problems exist and for which no local solutions exist.

(a) We consider the Fréchet space $E:=C^{\infty}([0,1], \mathbb{R})$ of smooth functions on the closed unit interval, and the continuous linear operator $D f:=f^{\prime}$ on $E$. We are asking for solutions of the initial value problem

$$
\dot{\gamma}(t)=D \gamma(t), \quad \gamma(0)=v_{0}, \quad \gamma: I \subseteq \mathbb{R} \rightarrow E .
$$

It follows from E. Borel's Theorem [B95] that there exist smooth functions $f_{+}$on $\left[1, \infty\left[\right.\right.$ and $f_{-}$on $\left.]-\infty, 0\right]$ such that

$$
f_{+}^{(j)}(1)=v_{0}^{(j)}(1) \quad \text { and } \quad f_{-}^{(j)}(0)=v_{0}^{(j)}(0) \quad \text { for } \quad j \in \mathbb{N}_{0} .
$$

Then

$$
h(x):= \begin{cases}f_{-}(x) & \text { for } x \leq 0, \\ v_{0}(x) & \text { for } 0 \leq x \leq 1, \\ f_{+}(x) & \text { for } x \geq 1\end{cases}
$$

is a smooth function on $\mathbb{R}$. The curve

$$
\gamma: \mathbb{R} \rightarrow E, \quad \gamma(t)(x):=h(t+x)
$$

satisfies $\gamma(0)=\left.h\right|_{[0,1]}=v_{0}$ and $\dot{\gamma}(t)(x)=h^{\prime}(t+x)=\gamma(t)^{\prime}(x)=(D \gamma(t))(x)$. It is clear that these solutions of (2.4) depend on the choice of the extension $h$ of $v_{0}$.

(b) We now consider the same problem on the space $F:=C^{\infty}(] 0,1[, \mathbb{R})$ with $D f=f^{\prime}$. Then the arguments under (a) imply that the corresponding initial value problem for $v_{0}(x):=\frac{1}{x-x^{2}}$ has no solution in any open interval containing 0 .

As we have just seen, there neither is a general result on local existence nor uniqueness of flows of smooth vector fields on Fréchet manifolds. Therefore the remarkable point of the following lemma is the uniqueness assertion.

Lemma 2.5. If $X \in \mathcal{V}(M)$ is a locally integrable vector field, then the following assertions hold:

(i) If $\Phi: \mathcal{D} \rightarrow M$ is a local flow on $M$ with $X^{\Phi}=X$, then the flow lines are integral curves of $X$.

(ii) For each open interval $I \subseteq \mathbb{R}$ containing 0 and $x \in M$, there is at most one integral curve $\gamma_{x}: I \rightarrow M$ of $X$.

(iii) Two local flows $\Phi, \Psi: \mathcal{D} \rightarrow M$ with $X^{\Phi}=X^{\Psi}$ coincide.

(iv) Through each point $x \in M$, there exists a unique maximal integral curve $\gamma_{x}: I_{x} \rightarrow M$ of $X$ with $0 \in I_{x}$ and $\gamma_{x}(0)=x$. All other integral curves $\gamma: I \rightarrow M$ of $X$ with $\gamma(0)=x$ are restrictions of $\gamma_{x}$.

Proof. (i) Let $\alpha_{x}: I_{x} \rightarrow M$ be a flow line and $s \in I_{x}$. For sufficiently small $t \in \mathbb{R}$, we then have

$$
\alpha_{x}(s+t)=\Phi_{s+t}(x)=\Phi_{t} \Phi_{s}(x)=\Phi_{t}\left(\alpha_{x}(s)\right),
$$

so that taking derivatives in $t=0$ leads to $\alpha_{x}^{\prime}(s)=X^{\Phi}\left(\alpha_{x}(s)\right)$.

(ii) Let $\gamma, \eta: I \rightarrow M$ be two integral curves of $X$ with $\gamma(0)=\eta(0)=p$. The continuity of the curves implies that

$$
0 \in J:=\{t \in I: \gamma(t)=\eta(t)\}
$$


is a closed subset of $I$. Suppose that there exists an element $0<t_{0} \in I \backslash J$. Then $s:=\sup J<t_{0}$ and $x:=\gamma(s)=\eta(s)$. We now consider the curve

$$
\widetilde{\gamma}(t):=\Phi_{-t}(\gamma(t+s)),
$$

defined on a small interval $I^{\prime}$ containing 0. Clearly, $\widetilde{\gamma}(0)=\gamma(s)=\alpha_{s}(x)$. Moreover, we obtain with (2.2)

$$
\begin{aligned}
\widetilde{\gamma}^{\prime}(t) & =-X(\widetilde{\gamma}(t))+T\left(\Phi_{-t}\right) \gamma^{\prime}(t+s)=-X(\widetilde{\gamma}(t))+X\left(\Phi_{-t}(\gamma(t+s))\right) \\
& =-X(\widetilde{\gamma}(t))+X(\widetilde{\gamma}(t))=0 .
\end{aligned}
$$

This implies that $\widetilde{\gamma}$ is constant (here we use the local convexity of $M$ ), so that $\Phi_{-t}(\gamma(t+s))=x$ for $t \in[0, \varepsilon]$ and some $\varepsilon>0$. If $\varepsilon$ is sufficiently small, then $\Phi_{-t}$ is defined on $\gamma(t+s)$ for $0 \leq t \leq \varepsilon$, and applying $\Phi_{t}$ on both sides leads to $\gamma(t+s)=$ $\alpha_{t}(x)$. The same argument shows that $\eta(t+s)=\alpha_{t}(x)$ for $0 \leq t$ sufficiently small. This contradicts the definition of $s$ and thus shows that $\sup J=\sup I$. We likewise obtain $\inf J=\inf I$, and therefore $J=I$.

(iii) follows immediately from (i) and (ii).

(iv) If $\gamma: I \rightarrow M$ and $\eta: J \rightarrow M$ are integral curves of $X$ with $\gamma(0)=\eta(0)=x$, then (ii) implies that $\left.\gamma\right|_{I \cap J}=\left.\eta\right|_{I \cap J}$, so that both curves combine to an integral curve on the open interval $I \cup J$.

Let $I_{x} \subseteq \mathbb{R}$ be the union of all open intervals $I_{j}$ containing 0 on which there exists an integral curve $\gamma_{j}: I_{j} \rightarrow M$ of $X$ with $\gamma_{j}(0)=x$. Then the preceding argument shows that

$$
\gamma(t):=\gamma_{j}(t) \quad \text { for } \quad t \in I_{j}
$$

defines an integral curve of $X$ on $I_{x}$, which is maximal by definition. The uniqueness of the maximal integral curve follows from its definition.

Theorem 2.6. Each locally integrable smooth vector field $X$ is the velocity field of a unique local flow defined by

$$
\mathcal{D}_{X}:=\bigcup_{x \in M} I_{x} \times\{x\} \quad \text { and } \quad \Phi_{t}(x):=\gamma_{x}(t) \quad \text { for } \quad(t, x) \in \mathcal{D}_{X},
$$

where $\gamma_{x}: I_{x} \rightarrow M$ is the unique maximal integral curve through $x \in M$.

Proof. If $(s, x),\left(t, \Phi_{s}(x)\right)$ and $(s+t, x) \in \mathcal{D}_{X}$, the relations

$$
\Phi_{s+t}(x)=\Phi_{t} \Phi_{s}(x) \quad \text { and } \quad I_{\Phi_{s}(x)}=I_{\gamma_{x}(s)}=I_{x}-s
$$

follow from the fact that both curves

$$
t \mapsto \Phi_{t+s}(x)=\gamma_{x}(t+s) \quad \text { and } \quad t \mapsto \Phi_{t} \Phi_{s}(x)=\gamma_{\Phi_{s}(x)}(t)
$$

are integral curves of $X$ with the initial value $\Phi_{s}(x)$, hence coincide.

We claim that all maps

$$
\Phi_{t}: M_{t}=\{m \in M:(t, m) \in \mathcal{D}\} \rightarrow M, \quad x \mapsto \Phi_{t}(x)
$$

are injective. In fact, if $p:=\Phi_{t}(x)=\Phi_{t}(y)$, then $\gamma_{x}(t)=\gamma_{y}(t)$, and on $[0, t]$ the curves $s \mapsto \gamma_{x}(t-s), \gamma_{y}(t-s)$ are integral curves of $-X$, starting in $p$. Hence Lemma 2.5)(ii) implies that they coincide in $s=t$, which means that $x=\gamma_{x}(0)=$ $\gamma_{y}(0)=y$. From this argument it further follows that $\Phi_{t}\left(M_{t}\right)=M_{-t}$ and $\Phi_{t}^{-1}=$ $\Phi_{-t}$. 
It remains to show that $\mathcal{D}_{X}$ is open and $\Phi$ smooth. The local integrability of $X$ provides for each $x \in M$ an open neighborhood $\mathcal{D}_{x}$ and some $\varepsilon_{x}>0$, as well as a smooth map

$$
\left.\varphi_{x}:\right]-\varepsilon_{x}, \varepsilon_{x}\left[\times \mathcal{D}_{x} \rightarrow M, \quad \varphi_{x}(t, y)=\gamma_{y}(t)=\Phi_{t}(y) .\right.
$$

Hence $]-\varepsilon_{x}, \varepsilon_{x}\left[\times \mathcal{D}_{x} \subseteq \mathcal{D}_{X}\right.$, and the restriction of $\Phi$ to this set is smooth. Therefore $\Phi$ is smooth on a neighborhood of $\{0\} \times M$ in $\mathcal{D}_{X}$.

Now let $J_{x}$ be the set of all $t \in\left[0, \infty\left[\right.\right.$, for which $\mathcal{D}_{X}$ contains a neighborhood of $[0, t] \times\{x\}$ on which $\Phi$ is smooth. The interval $J_{x}$ is open in $\mathbb{R}_{+}:=[0, \infty[$ by definition. We claim that $J_{x}=I_{x} \cap \mathbb{R}_{+}$. This entails that $\mathcal{D}_{X}$ is open because the same argument applies to $\left.\left.I_{x} \cap\right]-\infty, 0\right]$.

We assume the contrary and find a minimal $\tau \in I_{x} \cap \mathbb{R}_{+} \backslash J_{x}$, because this interval is closed. Put $p:=\Phi_{\tau}(x)$ and pick a product set $I \times W \subseteq \mathcal{D}_{X}$, where $W$ is an open neighborhood of $p$ and $I=]-2 \varepsilon, 2 \varepsilon$ [ a 0-neighborhood, such that $2 \varepsilon<\tau$ and $\Phi: I \times W \rightarrow M$ is smooth. By assumption, there exists an open neighborhood $V$ of $x$ such that $\Phi$ is smooth on $[0, \tau-\varepsilon] \times V \subseteq \mathcal{D}_{X}$. Then $\Phi_{\tau-\varepsilon}$ is smooth on $V$ and

$$
V^{\prime}:=\Phi_{\tau-\varepsilon}^{-1}\left(\Phi_{\varepsilon}^{-1}(W)\right) \cap V
$$

is a neighborhood of $[0, \tau+\varepsilon] \times\{x\}$ in $\mathcal{D}_{X}$. Further,

$$
V^{\prime}=\Phi_{\tau-\varepsilon}^{-1}\left(\Phi_{\varepsilon}^{-1}(W)\right) \cap V=\Phi_{\tau}^{-1}(W) \cap V,
$$

and $\Phi$ is smooth on $V^{\prime}$, because it is a composition of smooth maps:

$$
] \tau-2 \varepsilon, \tau+2 \varepsilon\left[\times V^{\prime} \rightarrow M, \quad(t, y) \mapsto \Phi_{t-\tau} \Phi_{\varepsilon} \Phi_{\tau-\varepsilon}(y) .\right.
$$

We thus arrive at the contradiction $\tau \in J_{x}$.

This completes the proof of the openness of $\mathcal{D}_{X}$ and the smoothness of $\Phi$. The uniqueness of the flow follows from the uniqueness of the integral curves (Lemma 2.5(ii)).

Remark 2.7. Let $\Phi^{X}: \mathcal{D}_{X} \rightarrow M$ be the maximal local flow of a locally integrable vector field $X$ on $M$. Let $M_{t}=\left\{x \in M:(t, x) \in \mathcal{D}_{X}\right\}$, and observe that this is an open subset of $M$. We have already seen in the proof of Theorem 2.6 above that the smooth maps $\Phi_{t}^{X}: M_{t} \rightarrow M$ are injective with $\Phi_{t}^{X}\left(M_{t}\right)=M_{-t}$ and $\left(\Phi_{t}^{X}\right)^{-1}=\Phi_{-t}^{X}$ on the image. It follows in particular that $\Phi_{t}^{X}\left(M_{t}\right)=M_{-t}$ is open and that $\Phi_{t}^{X}: M_{t} \rightarrow M_{-t}$ is a diffeomorphism whose inverse is $\Phi_{-t}^{X}$.

Remark 2.8. Suppose that $X \in \mathcal{V}(M)$ is locally integrable and that $\varphi: M \rightarrow N$ is a diffeomorphism. Then the vector field $Y:=\varphi_{*} X$ on $N$ is also locally integrable and its local flow satisfies

$$
\left.\Phi_{t}^{Y} \circ \varphi\right|_{M_{t}}=\left.\varphi \circ \Phi_{t}^{X}\right|_{M_{t}} .
$$

Proposition 2.9. Let $V$ be a locally convex vector space and $D: V \rightarrow V$ be a continuous linear map. If the corresponding smooth vector field $X(v)=D v$ is locally integrable, then the corresponding local flow is global.

Proof. Suppose that $X$ is locally integrable. Then the domain $\mathcal{D} \subseteq \mathbb{R} \times V$ of its local flow contains a neighborhood $[-\varepsilon, \varepsilon] \times U_{V}$ of $(0,0)$. The linearity of the vector field further implies that each subset $V_{t}, t \in \mathbb{R}$, is a linear subspace of $V$, hence $V_{t}=V$ whenever $V_{t} \neq \emptyset$. We conclude that $[-\varepsilon, \varepsilon] \times V \subseteq \mathcal{D}$, and this implies that the flow it global. 


\section{LIE DERIVATIVES}

In this section we take a closer look at the interaction of local flows and vector fields. Let $X \in \mathcal{V}(M)$ be locally integrable and let $\Phi^{X}: \mathcal{D}_{X} \rightarrow M$ be its maximal local flow. For $f \in C^{\infty}(M)$ and $t \in \mathbb{R}$, we set

$$
\left(\Phi_{t}^{X}\right)^{*} f:=f \circ \Phi_{t}^{X} \in C^{\infty}\left(M_{t}\right) .
$$

Then

$$
\lim _{t \rightarrow 0} \frac{1}{t}\left(\left(\Phi_{t}^{X}\right)^{*} f-f\right)=\mathrm{d} f(X)=\mathcal{L}_{X} f \in C^{\infty}(M) .
$$

For a second vector field $Y \in \mathcal{V}(M)$, we define a smooth vector field on the open subset $M_{-t} \subseteq M$ by

$$
\left(\Phi_{t}^{X}\right)_{*} Y:=T\left(\Phi_{t}^{X}\right) \circ Y \circ \Phi_{-t}^{X}=T\left(\Phi_{t}^{X}\right) \circ Y \circ\left(\Phi_{t}^{X}\right)^{-1}
$$

(cf. Remark 2.7) and define the Lie derivative by

$$
\mathcal{L}_{X} Y:=\lim _{t \rightarrow 0} \frac{1}{t}\left(\left(\Phi_{-t}^{X}\right)_{*} Y-Y\right)=\left.\frac{d}{d t}\right|_{t=0}\left(\Phi_{-t}^{X}\right)_{*} Y,
$$

which is defined on all of $M$ since, for each $p \in M$, the vector $\left(\left(\Phi_{t}^{X}\right)_{*} Y\right)(p)$ is defined for sufficiently small $t$ and depends smoothly on $t$.

Theorem 3.1. $\mathcal{L}_{X} Y=[X, Y]$ for $X, Y \in \mathcal{V}(M)$.

Proof. Fix $p \in M$. It suffices to show that $\mathcal{L}_{X} Y$ and $[X, Y]$ coincide in $p$. We may therefore work in a local chart, hence assume that $M=U$ is an open subset of some locally convex space $E$.

Identifying vector fields with smooth $E$-valued functions, we have

$$
[X, Y](x)=\mathrm{d} Y(x) X(x)-\mathrm{d} X(x) Y(x) \quad \text { for } \quad x \in U .
$$

On the other hand,

$$
\begin{aligned}
\left(\left(\Phi_{-t}^{X}\right)_{*} Y\right)(x) & =T\left(\Phi_{-t}^{X}\right) \circ Y \circ \Phi_{t}^{X}(x)=\mathrm{d}\left(\Phi_{-t}^{X}\right)\left(\Phi_{t}^{X}(x)\right) Y\left(\Phi_{t}^{X}(x)\right) \\
& =\left(\mathrm{d}\left(\Phi_{t}^{X}\right)(x)\right)^{-1} Y\left(\Phi_{t}^{X}(x)\right) .
\end{aligned}
$$

To calculate the derivative of this expression with respect to $t$, we first observe that it does not matter if we first take derivatives with respect to $t$ and then with respect to $x$ or vice versa. This leads to

$$
\left.\frac{d}{d t}\right|_{t=0} \mathrm{~d}\left(\Phi_{t}^{X}\right)(x)=\mathrm{d}\left(\left.\frac{d}{d t}\right|_{t=0} \Phi_{t}^{X}\right)(x)=\mathrm{d} X(x) .
$$

Next we note that for any curve $\alpha:[-\varepsilon, \varepsilon] \rightarrow \mathrm{GL}(V)$ with $\alpha(0)=\mathbf{1}$ for which the associated map

$$
\widehat{\alpha}:[-\varepsilon, \varepsilon] \times V \rightarrow V \times V, \quad \widehat{\alpha}(t, v):=\left(\alpha(t) v, \alpha(t)^{-1} v\right)
$$

is smooth, we have

$$
\left(\alpha^{-1}\right)^{\prime}(t)=-\alpha(t)^{-1} \alpha^{\prime}(t) \alpha(t)^{-1},
$$

and in particular $\left(\alpha^{-1}\right)^{\prime}(0)=-\alpha^{\prime}(0)$. Combining all this, we obtain with the Product Rule

$$
\mathcal{L}_{X}(Y)(x)=-\mathrm{d} X(x) Y(x)+\mathrm{d} Y(x) X(x)=[X, Y](x) .
$$


Example 3.2. If $V=C_{c}^{\infty}(] 0,1[)$ and $D f=f^{\prime}$, then for every $f \in V$, there exists a smooth integral curve $\gamma:[-\varepsilon, \varepsilon] \rightarrow V$ with $\dot{\gamma}(t)=D \gamma(t)$, given by $\gamma(t)(s)=f(t+s)$ for $\operatorname{supp}(f)-t \subseteq] 0,1[$, but the corresponding linear vector field $\mathbf{x}(v)=D v$ is not locally integrable since the corresponding flow is not defined on a neighborhood of $(0,0)$ in $\mathbb{R} \times V$ (cf. Proposition 2.9) .

Definition 3.3. A locally convex Lie algebra $\mathfrak{g}$ is call ad-integrable if all linear vector fields $X_{x}(y):=[x, y]$ generate global flows. If $\mathfrak{g}$ is ad-integrable, then we set

$$
e^{\operatorname{ad} x}:=\Phi_{1}^{X_{x}} \quad \text { for } \quad x \in \mathfrak{g} .
$$

Lemma 3.4. Let $\mathfrak{g}$ be a locally convex ad-integrable Lie algebra. Let $\beta: \mathfrak{g} \rightarrow \mathcal{V}(M)$ be a homomorphism of Lie algebras for which the corresponding map

$$
\widehat{\beta}: \mathfrak{g} \times M \rightarrow T M, \quad(x, m) \mapsto \beta(x)(m)
$$

is smooth. Suppose that $\beta(x)$ is locally integrable and write $\Phi^{x}$ for the corresponding local flow on $M$. Then

$$
\left(\Phi_{-t}^{x}\right)_{*} \beta(y)=\left.\beta\left(e^{t \operatorname{ad} x} y\right)\right|_{M_{t}} \quad \text { for } \quad y \in \mathfrak{g}, t \in \mathbb{R} .
$$

Proof. Pick $m \in M$ and assume that $\Phi_{-t}^{x}(m)$ is defined in $t_{0}$. For the curve

$$
\gamma(t):=\left(\left(\Phi_{t}^{x}\right)_{*} \beta\left(e^{t \operatorname{ad} x} y\right)\right)(m)=T\left(\Phi_{t}^{x}\right) \beta\left(e^{t \operatorname{ad} x} y\right)\left(\Phi_{-t}^{x}(m)\right)
$$

our smoothness assumption on $\beta$ implies with Theorem 3.1 that

$$
\begin{aligned}
\gamma^{\prime}\left(t_{0}\right) & =\left.\frac{d}{d t}\right|_{t=t_{0}}\left(\left(\Phi_{t}^{x}\right)_{*} \beta\left(e^{t_{0} \text { ad } x} y\right)\right)(m)+\left.\frac{d}{d t}\right|_{t=t_{0}}\left(\left(\Phi_{t_{0}}^{x}\right)_{*} \beta\left(e^{t \operatorname{ad} x} y\right)\right)(m) \\
& =\left(\left(\Phi_{t_{0}}^{x}\right)_{*} \mathcal{L}_{-\beta(x)} \beta\left(e^{t_{0} \text { ad } x} y\right)\right)(m)+\left(\left(\Phi_{t_{0}}^{x}\right)_{*} \beta\left(\left[x, e^{t_{0} \text { ad } x} y\right]\right)\right)(m) \\
& =\left(\left(\Phi_{t_{0}}^{x}\right)_{*}\left(\left[-\beta(x), \beta\left(e^{t_{0} \text { ad } x} y\right)\right]+\beta\left(\left[x, e^{t_{0} \text { ad } x} y\right]\right)\right)\right)(m)=0 .
\end{aligned}
$$

We conclude that $\gamma$ is constant, and hence that, whenever $\Phi_{-t}^{x}(m)$ is defined, we have

This leads to

$$
T\left(\Phi_{t}^{x}\right) \beta\left(e^{t \operatorname{ad} x} y\right)\left(\Phi_{-t}^{x}(m)\right)=\beta(y)(m)
$$

$$
\beta\left(e^{t \operatorname{ad} x} y\right)(m)=T\left(\Phi_{-t}^{x}\right) \beta(y)\left(\Phi_{t}^{x}(m)\right)=\left(\left(\Phi_{-t}^{x}\right)_{*} \beta(y)\right)(m) \quad \text { for } \quad m \in M_{t},
$$

so that (3.1) is proved.

\section{VECTOR FIELDS AND POSITIVE DEFINITE KERNELS}

In this section $M$ is a smooth locally convex manifold and we study the interaction between a smooth positive definite kernel $K: M \times M \rightarrow \mathbb{C}$ and vector fields. Our main result is the Geometric Fröhlich Theorem (Theorem 4.6) which implies in particular that for a locally integrable smooth vector field $X \in \mathcal{V}(M)$ the Lie derivative defines a selfadjoint operator on the corresponding reproducing kernel space $\mathcal{H}_{K}$.

Definition 4.1. Let $K \in C^{\infty}(M \times M, \mathbb{C})$ be a smooth positive definite kernel. A vector field $X \in \mathcal{V}(M)$ is said to be $K$-symmetric if

$$
\mathcal{L}_{X}^{1} K=\mathcal{L}_{X}^{2} K
$$

and $K$-skew-symmetric if

$$
\mathcal{L}_{X}^{1} K=-\mathcal{L}_{X}^{2} K
$$


Here the superscripts indicate whether the Lie derivative acts on the first or the second argument.

In the following we assume that $K$ is a smooth positive definite kernel on $M$ and write $\mathcal{H}_{K} \subseteq \mathbb{C}^{M}$ for the corresponding reproducing kernel Hilbert space (see Appendix A for details). Then the functions $K_{m}:=K(\cdot, m), m \in M$, span a dense subspace $\mathcal{H}_{K}^{0}$ and we have

$$
f(m)=\left\langle f, K_{m}\right\rangle \quad \text { for } \quad f \in \mathcal{H}_{K}, m \in M
$$

(cf. Definition A.1). According to [Ne10, Thm. 7.1], the smoothness of $K$ implies that the map

$$
M \rightarrow \mathcal{H}_{K}, \quad m \rightarrow K_{m},
$$

is smooth, so that $\varphi(m)=\left\langle\varphi, K_{m}\right\rangle$ for $\varphi \in \mathcal{H}_{K}$ implies that $\mathcal{H}_{K} \subseteq C^{\infty}(M, \mathbb{C})$. The Lie derivative defines a representation of $\mathcal{V}(M)$ on $C^{\infty}(M, \mathbb{C}) \supseteq \mathcal{H}_{K}$. For every smooth vector field $X \in \mathcal{V}(M)$, we therefore define

$$
\mathcal{D}_{X}:=\left\{\varphi \in \mathcal{H}_{K}: \mathcal{L}_{X} \varphi \in \mathcal{H}_{K}\right\}, \quad \mathcal{L}_{X}^{K}:=\left.\mathcal{L}_{X}\right|_{\mathcal{D}_{X}}: \mathcal{D}_{X} \rightarrow \mathcal{H}_{K}
$$

Lemma 4.2. If $\gamma:[a, b] \rightarrow M$ is an integral curve of $X$ and $\mathcal{L}_{X}^{1} K=\varepsilon \mathcal{L}_{X}^{2} K$, $\varepsilon \in\{ \pm 1\}$, then the curve $\eta(t):=K_{\gamma(t)}$ in $\mathcal{H}_{K}$ is smooth, contained in $\mathcal{D}_{X}$, and satisfies the differential equation

$$
\eta^{\prime}(t)=\varepsilon \mathcal{L}_{X}^{K} \eta(t)
$$

Proof. The smoothness of the map $M \rightarrow \mathcal{H}_{K}, m \mapsto K_{m}$, and the smoothness of $\gamma$ imply that $\eta$ is smooth. We further have, for $m \in M$,

$$
\begin{aligned}
\eta^{\prime}(t)(m) & =\left\langle\eta^{\prime}(t), K_{m}\right\rangle=\frac{d}{d t} K(m, \gamma(t))=\mathcal{L}_{X}^{2} K(m, \gamma(t)) \\
& =\varepsilon \mathcal{L}_{X}^{1} K(m, \gamma(t))=\varepsilon \mathcal{L}_{X} K_{\gamma(t)}(m) .
\end{aligned}
$$

This implies (4.1). We conclude in particular that $\eta(t) \in \mathcal{D}_{X}$, and the assertion follows.

Proposition 4.3. Suppose that $X \in \mathcal{V}(M)$ satisfies $\mathcal{L}_{X}^{1} K=\varepsilon \mathcal{L}_{X}^{2} K$ for $\varepsilon \in\{ \pm 1\}$ and that integral curves through every $m \in M$ exist. Then the following assertions hold:

(i) $\mathcal{H}_{K}^{0} \subseteq \mathcal{D}_{X}$.

(ii) If $X$ is symmetric (resp. skew-symmetric), then $\left.\mathcal{L}_{X}^{K}\right|_{\mathcal{H}_{k}^{0}}$ is a symmetric (resp. skew-symmetric) operator.

(iii) $\mathcal{L}_{X}^{K}$ is a closed operator.

(iv) If $\left.\mathcal{L}_{X}^{K}\right|_{\mathcal{H}_{k}^{0}}$ is essentially selfadjoint (resp. skew-adjoint), then $\mathcal{L}_{X}^{K}=\overline{\left.\mathcal{L}_{X}^{K}\right|_{\mathcal{H}_{k}^{0}}}$.

Proof. (i) The relation $\mathcal{H}_{K}^{0} \subseteq \mathcal{D}_{X}$ follows directly from Lemma 4.2 .

(ii) Since $K(m, n)=\overline{K(n, m)}$, we have

$$
\begin{aligned}
\left\langle\mathcal{L}_{X} K_{m}, K_{n}\right\rangle & =\mathcal{L}_{X}^{1} K(n, m)=\varepsilon \mathcal{L}_{X}^{2} K(n, m)=\varepsilon \overline{\mathcal{L}_{X}^{1} K(m, n)} \\
& =\varepsilon \overline{\left\langle\mathcal{L}_{X} K_{n}, K_{m}\right\rangle}=\varepsilon\left\langle K_{m}, \mathcal{L}_{X} K_{n}\right\rangle .
\end{aligned}
$$

(iii) To see that $\mathcal{L}_{X}^{K}$ is closed, assume that $\left(f_{n}, \mathcal{L}_{X}^{K} f_{n}\right) \rightarrow(f, g)$ in $\mathcal{H}_{K} \times \mathcal{H}_{K}$. Then we have, for every $m \in M$, any integral curve $\gamma_{m}$ through $m$, and $\varphi \in \mathcal{H}_{K}$, the relation

$$
\left(\mathcal{L}_{X} \varphi\right)(m)=\left.\frac{d}{d t}\right|_{t=0} \varphi \circ \gamma_{m}=\left.\frac{d}{d t}\right|_{t=0}\left\langle\varphi, K_{\gamma_{m}}\right\rangle=\varepsilon\left\langle\varphi, \mathcal{L}_{X}^{K} K_{m}\right\rangle
$$


In particular, $f_{n} \rightarrow f$ implies that $\mathcal{L}_{X} f_{n} \rightarrow \mathcal{L}_{X} f$ pointwise on $M$, and since we also have $\left(\mathcal{L}_{X}^{K} f_{n}\right)(m)=\left\langle\mathcal{L}_{X}^{K} f_{n}, K_{m}\right\rangle \rightarrow\left\langle g, K_{m}\right\rangle=g(m)$, it follows that $g=\mathcal{L}_{X} f$. This implies that $f \in \mathcal{D}_{X}$ and $g=\mathcal{L}_{X}^{K} f$.

(iv) Let $T=\overline{\left.\mathcal{L}_{X}^{K}\right|_{\mathcal{H}_{k}^{0}}}$. Then our assumption implies $T=\varepsilon\left(\left.\mathcal{L}_{X}^{K}\right|_{\mathcal{H}_{k}^{0}}\right)^{*}$. Since $\mathcal{L}_{X}^{K}$ is closed, we have $T \subseteq \mathcal{L}_{X}^{K}$. Conversely, (4.2) shows that $\mathcal{L}_{X}^{K} \subseteq \varepsilon\left(\left.\mathcal{L}_{X}^{K}\right|_{\mathcal{H}_{K}^{0}}\right)^{*}$. Hence $\mathcal{L}_{X}^{K}=T$.

Lemma 4.4. Suppose that $X \in \mathcal{V}(M)$ has the property that there exists a smooth $\mathbb{R}$-action $\sigma: \mathbb{R} \times M \rightarrow M$ for which the orbit curves $t \mapsto \sigma_{t}(m):=\sigma(t, m)$ are integral curves of $X$. If $X$ is $K$-skew-symmetric, then

$$
U_{t} \varphi:=\varphi \circ \sigma_{t}
$$

defines a continuous unitary representation on $\mathcal{H}_{K}$ with

$$
U_{t} K_{m}=K_{\sigma_{-t}(m)} \text {. }
$$

In particular, $\mathcal{H}_{K}^{0}$ consists of smooth vectors for $U$. Moreover, the infinitesimal generator of $U$ coincides with $\mathcal{L}_{X}^{K}$ and $\mathcal{H}_{K}^{0}$ is a core for $\mathcal{L}_{X}^{K}$.

Proof. The invariance of $K$ under the flow $\sigma$ follows immediately from the $K$-skewsymmetry of $K$, which shows that, for $m, n \in M$, the functions $t \rightarrow K\left(\sigma_{t}(m), \sigma_{t}(n)\right)$ are constant. This implies that $K_{m} \circ \sigma_{t}=K_{\sigma_{-t}(m)}$, which is (4.3). The invariance of $\mathcal{H}_{K}$ under $\sigma$ and that $U$ is a unitary representation follows from [Ne00, Prop. II.4.9]. As $\mathcal{H}_{K}^{0}$ is $U$-invariant, Stone's Theorem implies that the infinitesimal generator $T$ of $U$ coincides with the closure of $\left.T\right|_{\mathcal{H}_{K}^{0}}$. Since on $\mathcal{D}(T)$, and in particular on $\mathcal{H}_{K}^{0}$, $T$ and $\mathcal{L}_{X}^{K}$ coincide, the last assertion follows from Proposition 4.3(iv).

Below we recall Fröhlich's Theorem on unbounded symmetric semigroups as it is stated in [Fro80, Cor. 1.2] (see also [MN12]). Actually, Fröhlich assumes that the Hilbert space $\mathcal{H}$ is separable, but this is not necessary for the conclusion. Replacing the assumption of weak measurability by weak continuity, all arguments in Fro80. work for non-separable spaces as well.

Theorem 4.5 (Fröhlich). Let $H$ be a symmetric operator defined on the dense subspace $\mathcal{D}$ of the Hilbert space $\mathcal{H}$. Suppose that, for every $v \in \mathcal{D}$, there exists an $\varepsilon_{v}>0$ and a curve $\left.\varphi:\right] 0, \varepsilon_{v}[\rightarrow \mathcal{D}$ satisfying

$$
\dot{\varphi}(t)=H \varphi(t) \quad \text { and } \quad \lim _{t \rightarrow 0} \varphi(t)=v .
$$

Then the operator $H$ is essentially selfadjoint and $\varphi(t)=e^{t \bar{H}} v$ in the sense of spectral calculus.

In MN12, Rem. 2.6] we applied Fröhlich's Theorem to the action of the Lie derivative on a Hilbert space with a smooth reproducing kernel. This leads to the following theorem.

Theorem 4.6 (Geometric Fröhlich Theorem). Let $M$ be a locally convex manifold and let $K$ be a smooth positive definite kernel. If $X$ is a $K$-symmetric vector field on $M$ with the property that, for every $m \in M$, there exists an integral curve $\gamma_{m}:\left[0, \varepsilon_{m}\right] \rightarrow M$ starting in $m$, then $\left.\mathcal{L}_{X}\right|_{\mathcal{H}_{K}^{0}}$ is an essentially selfadjoint operator $\mathcal{H}_{K}^{0} \rightarrow \mathcal{H}_{K}$ whose closure coincides with $\mathcal{L}_{X}^{K}$. For $0 \leq t \leq \varepsilon_{m}$, we have

$$
e^{t \mathcal{L}_{X}^{K}} K_{m}=K_{\gamma_{m}(t)}
$$


Proof. First we recall that the $K$-symmetry of $X$ implies that $H:=\left.\mathcal{L}_{X}^{K}\right|_{\mathcal{H}_{K}^{0}}$ is a symmetric operator (Proposition 4.3(ii)). Lemma 4.2 further implies that, for every integral curve $\gamma$ of $X$, the curve $\eta(t):=K_{\gamma(t)}$ in $\mathcal{H}_{K}$ satisfies the ODE $\eta^{\prime}(t)=H \eta(t)$. Therefore Theorem 4.5 applies and $\mathcal{L}_{X}^{K}=\bar{H}$ follows from Proposition 4.3(iv).

Example 4.7. The simplest but typical class of examples where Fröhlich's Theorem applies arise from smooth functions $\varphi:] a, b[\rightarrow \mathbb{C}$ on an open interval in $\mathbb{R}$ for which the kernel $K(x, y):=\varphi\left(\frac{x+y}{2}\right)$ is positive definite. Then the vector field $X=\frac{\partial}{\partial t}$ satisfies $\mathcal{L}_{X} f=f^{\prime}$ on $\mathcal{H}_{K}^{0}$, so that the operator $D f:=f^{\prime}$ on its maximal domain in $\mathcal{H}_{K}$ is selfadjoint by the preceding theorem.

To see the corresponding unitary one-parameter group $U_{t}:=e^{i t D}$, one can use Sh84 to see that the function $\varphi$ extends analytically to the strip

$$
S:=\{z \in \mathbb{C}: a<\operatorname{Re} z<b\},
$$

so that $K^{\mathbb{C}}(z, w):=\varphi\left(\frac{z+\bar{w}}{2}\right)$ provides an extension of the kernel $K$ to $S \times S$. On the corresponding Hilbert space $\mathcal{H}_{K^{\mathbb{C}}} \subseteq \mathcal{O}(S)$, we then have $\left(U_{t} f\right)(z)=f(z+i t)$.

Example 4.8. For later use we record here how Fröhlich's Theorem applies in the linear case. Let $V$ be a locally convex space and $\langle\cdot, \cdot\rangle: V \times V \rightarrow \mathbb{C}$ be a continuous positive semidefinite hermitian form. Then $K(v, w)=\langle w, v\rangle$ is a positive definite kernel and $\mathcal{H}_{K}$ is identified with a subspace of the space $V^{\sharp}$ of antilinear continuous functionals. The continuity of the kernel $K$ implies that the antilinear map $V \rightarrow \mathcal{H}_{K}, v \mapsto K_{v}$ is continuous. For any continuous operator $L: V \rightarrow V$, the formula

$$
L \lambda:=-\lambda \circ L
$$

defines by restriction to $\mathcal{D}_{L}:=\left\{\lambda \in V^{\sharp}: L \lambda \in \mathcal{H}_{K}\right\}$ an unbounded closed operator $L^{K}: \mathcal{D}_{L} \rightarrow \mathcal{H}_{K}$. If there exists $L^{*}: V \rightarrow V$ with

$$
\langle v, L w\rangle=\left\langle L^{*} v, w\right\rangle \quad \text { for } \quad v, w \in V,
$$

then

$$
L K_{v}=K_{-L^{*} v} \quad \text { for } \quad v \in V .
$$

We can now formulate the following corollary to Theorem 4.5 ;

Corollary 4.9. Let $L: V \rightarrow V$ be a symmetric linear operator with the property that, for every $v \in V$, there exists a curve $\gamma_{v}:\left[0, \varepsilon_{v}\right] \rightarrow V$ starting in $v$ and satisfying the differential equation

$$
\gamma_{v}^{\prime}(t)=L \gamma_{v}(t)
$$

Then $\left.L^{K}\right|_{\mathcal{H}_{K}^{0}}$ is an essentially selfadjoint operator whose closure coincides with $L^{K}$. For $0 \leq t \leq \varepsilon_{v}$, we have

$$
e^{-t L^{K}} K_{v}=K_{\gamma_{v}(t)}
$$

\section{INTEGRABILITY FOR REPRODUCING KERNEL SPACES}

In this section we study Lie algebra actions on the locally convex manifold $M$ that are compatible with the kernel $K$. Our first main result is Theorem 5.12 which provides a sufficient condition for the Lie algebra representation of $\mathfrak{g}^{c}$ coming from an action of $\mathfrak{g}$ on $\mathcal{H}_{K}$ by Lie derivatives to integrate to a unitary representation of the corresponding simply connected Lie group $G^{c}$. Applying this result to open subsemigroups of Lie groups, we further obtain an interesting generalization of the 
Lüscher-Mack Theorem for semigroups that need not possess a polar decomposition.

\subsection{Smooth right actions and compatible kernels.}

Definition 5.1. Let $\mathfrak{g}$ be a Lie algebra and $\tau$ be an involutive automorphism of $\mathfrak{g}$. The pair $(\mathfrak{g}, \tau)$ is called a symmetric Lie algebra. If $\mathfrak{h}:=\operatorname{ker}(\tau-\mathbf{1})$ and $\mathfrak{q}=\operatorname{ker}(\tau+\mathbf{1})$, then $[\mathfrak{h}, \mathfrak{h}] \subseteq \mathfrak{h},[\mathfrak{q}, \mathfrak{q}] \subseteq \mathfrak{h}$ and $[\mathfrak{h}, \mathfrak{q}] \subseteq \mathfrak{q}$. It follows that $\mathfrak{g}^{c}:=\mathfrak{h}+i \mathfrak{q}$ is a Lie algebra. It is called the Cartan dual of $\mathfrak{g}=\mathfrak{h}+\mathfrak{q}$. Extending $\tau$ to a complex linear automorphism of $\mathfrak{g}_{\mathbb{C}}$ and then restricting to $\mathfrak{g}^{c}$ shows that $\left(\mathfrak{g}^{c}, \tau\right)$ is also a symmetric Lie algebra.

Example 5.2. Let $d \in \mathbb{N}$ and $p, q \in \mathbb{N}_{0}$ such that $p+q=d$. Let

$$
I_{p, q}=\left(\begin{array}{cc}
-I_{p} & 0 \\
0 & I_{q}
\end{array}\right) .
$$

On $G=\mathbb{R}^{d} \rtimes \mathrm{O}_{d}(\mathbb{R})$ we consider the involution

$$
\tau(x, a)=\left(I_{p, q} x, I_{p, q} a I_{p, q}\right) .
$$

Then

$$
\mathfrak{q}=\left(\mathbb{R}^{p} \oplus 0_{q}\right) \oplus\left\{\left(\begin{array}{cc}
0 & X \\
-X^{\top} & 0
\end{array}\right): X \in M_{p, q}\right\}
$$

It is then easy to see that

$$
\mathfrak{g}^{c} \simeq \mathbb{R}^{p, q} \rtimes \mathfrak{o}_{p, q}(\mathbb{R}) .
$$

Hence $G^{c}$ is locally isomorphic to $\mathbb{R}^{p, q} \rtimes \mathrm{O}_{p, q}(\mathbb{R})$. Here $\mathbb{R}^{p, q} \cong i \mathbb{R}^{p} \oplus \mathbb{R}^{q}$ stands for the vector space $\mathbb{R}^{d}$ with the bilinear form

$$
\beta_{p, q}(x, y)=x_{1} y_{1}+\ldots+x_{p} y_{p}-x_{p+1} y_{p+1}-\ldots-x_{n} y_{n} .
$$

Definition 5.3. Let $(\mathfrak{g}, \tau)$ be a symmetric Lie algebra, and let $\beta: \mathfrak{g} \rightarrow \mathcal{V}(M)$ be a homomorphism. A smooth positive definite kernel $K \in C^{\infty}(M \times M, \mathbb{C})$ is said to be $\beta$-compatible if the vector fields in $\beta(\mathfrak{h})$ are $K$-skew-symmetric and the vector fields in $\beta(\mathfrak{q})$ are $K$-symmetric.

Let $H$ be a connected Lie group with Lie algebra $\mathfrak{h}$.

Definition 5.4. A smooth right action of the pair $(\mathfrak{g}, H)$ on a (locally convex) manifold $M$ is a pair $(\beta, \sigma)$, where

(a) $\sigma: M \times H \rightarrow M$ is a smooth right action,

(b) and $\beta: \mathfrak{g} \rightarrow \mathcal{V}(M)$ is a homomorphism of Lie algebras for which

$$
\widehat{\beta}: \mathfrak{g} \times M \rightarrow T M, \quad(x, m) \mapsto \beta(x)(m)
$$

is smooth,

(c) $\dot{\sigma}(x)=\beta(x)$ for $x \in \mathfrak{h}$,

(d) each vector field $\beta(x), x \in \mathfrak{q}$, is locally integrable.

Remark 5.5. In view of Lemma 3.4, conditions (a)-(d) above imply that

$$
\left(\sigma_{\exp x}\right)_{*} \beta(y)=\beta\left(e^{-\operatorname{ad} x} y\right) \quad \text { for } \quad x \in \mathfrak{h}, y \in \mathfrak{g} .
$$


In the following we consider a smooth right action $(\beta, \sigma)$ of $(\mathfrak{g}, H)$ on $M$ together with a smooth $\beta(\mathfrak{g})$-compatible positive definite kernel $K$ on $M \times M$. For $x \in \mathfrak{g}$, we abbreviate $\mathcal{L}_{x}:=\mathcal{L}_{\beta(x)}^{K}$ for the maximal restriction of the Lie derivatives to $\mathcal{D}_{x}:=\mathcal{D}_{\beta(x)}$ and we extend this definition to $\mathfrak{g}_{\mathbb{C}}$ in an obvious fashion. We moreover consider the subspaces

$$
\mathcal{D}^{1}:=\bigcap_{x \in \mathfrak{g}} \mathcal{D}_{x}=\left\{\varphi \in \mathcal{H}_{K}:(\forall x \in \mathfrak{g}) \mathcal{L}_{\beta(x)} \varphi \in \mathcal{H}_{K}\right\}
$$

and

$$
\mathcal{D}:=\left\{\varphi \in \mathcal{H}_{K}:(\forall n \in \mathbb{N})\left(\forall x_{1}, \ldots, x_{n} \in \mathfrak{g}\right) \mathcal{L}_{\beta\left(x_{1}\right)} \cdots \mathcal{L}_{\beta\left(x_{n}\right)} \varphi \in \mathcal{H}_{K}\right\}
$$

Then

$$
\alpha: \mathfrak{g}_{\mathbb{C}} \rightarrow \operatorname{End}(\mathcal{D}),\left.x \mapsto \mathcal{L}_{x}\right|_{\mathcal{D}}
$$

defines a Lie algebra representation such that $\mathfrak{g}^{c}$ acts by skew-symmetric operators. Our main goal in this section is to prove integrability of $\left.\alpha\right|_{\mathfrak{g}^{c}}$.

First we show that $\mathcal{D}$ is a dense subspace of $\mathcal{H}_{K}$.

Proposition 5.6. We have $\mathcal{H}_{K}^{0} \subseteq \mathcal{D}$. In particular $\mathcal{D}$ is dense in $\mathcal{H}$.

Proof. Let $x_{1}, \ldots, x_{n} \in \mathfrak{h} \cup \mathfrak{q}, m \in M$, and choose $\varepsilon>0$ so small that we have a smooth map

$$
\gamma:[0, \varepsilon]^{n} \rightarrow M, \gamma\left(t_{1}, \ldots, t_{n}\right)=\Phi_{t_{1}}^{\beta\left(x_{1}\right)} \circ \cdots \circ \Phi_{t_{n}}^{\beta\left(x_{n}\right)}(m) .
$$

Then the curves

$$
t \mapsto \gamma\left(0, \ldots, 0, t, t_{k+1}, \ldots, t_{n}\right)
$$

are integral curves of $\beta\left(x_{k}\right)$. Let $\eta\left(t_{1}, \ldots, t_{n}\right):=K_{\gamma\left(t_{1}, \ldots, t_{n}\right)}$. Then $\eta:[0, \varepsilon]^{n} \rightarrow \mathcal{H}_{K}$ is smooth and Lemma 4.2 implies that

$$
\frac{\partial}{\partial t_{k}} \eta\left(0, \ldots, 0, t_{k}, \ldots, t_{n}\right)=\varepsilon_{k} \mathcal{L}_{x_{k}} \eta\left(0, \ldots, 0, t_{k}, \ldots, t_{n}\right),
$$

where $\mathcal{L}_{\beta\left(x_{k}\right)}^{1} K=\varepsilon_{k} \mathcal{L}_{\beta\left(x_{k}\right)}^{2} K$. We conclude with the closedness of the operators $\mathcal{L}_{\beta\left(x_{j}\right)}^{K}$ (Proposition 4.3) that $K_{\gamma(0)} \in \mathcal{D}$ with

$$
\frac{\partial^{n} \eta}{\partial t_{1} \cdots \partial t_{n}}(0)=\left(\varepsilon_{1} \cdots \varepsilon_{n}\right) \mathcal{L}_{x_{1}} \cdots \mathcal{L}_{x_{n}} K_{\gamma(0)}
$$

This shows that $\mathcal{H}_{K}^{0} \subseteq \mathcal{D}$.

From Theorem 4.6] and Proposition [5.6, we obtain:

Corollary 5.7. For $x \in \mathfrak{q}$, the operator $\mathcal{L}_{x}$ is selfadjoint and $\mathcal{H}_{K}^{0}$, and hence also $\mathcal{D}$, is a core for $\mathcal{L}_{x}$.

The following lemma follows from Lemma 4.4.

Lemma 5.8. The reproducing kernel $K$ is $H$-invariant and

$$
\pi^{H}(h) \varphi:=\varphi \circ \sigma_{h}
$$

defines a continuous unitary representation $\left(\pi^{H}, \mathcal{H}_{K}\right)$ of $H$, for which

$$
\pi^{H}(h) K_{m}=K_{m . h^{-1}} \quad \text { for } \quad m \in M, h \in H .
$$

In particular, $\mathcal{H}_{K}^{0} \subseteq \mathcal{H}^{\infty}\left(\pi^{H}\right)$. For $x \in \mathfrak{h}$, the infinitesimal generator of the unitary one-parameter group $\pi_{x}^{H}(t):=\pi^{H}(\exp t x)$ is given by $\mathcal{L}_{x}=\overline{\mathrm{d} \pi^{H}}(x)$. Moreover, $\mathcal{H}_{K}^{0}$, and hence $\mathcal{D}$ is a core for $\mathcal{L}_{x}$. 
5.2. The Integrability Theorem. The proof of the Integrability Theorem will be based on the following result.

Theorem 5.9 (Mer11). Let $G^{c}$ be a simply connected Banach-Lie group with Lie algebra $\mathfrak{g}^{c}$. Assume that $\mathfrak{g}^{c}=\mathfrak{a}_{1} \oplus \mathfrak{a}_{2}$ where $\mathfrak{a}_{1}$ and $\mathfrak{a}_{2}$ are closed subspaces. Let $\alpha$ be a strongly continuous representation of $\mathfrak{g}$ on a dense subspace $\mathcal{D}$ of a Hilbert space $\mathcal{H}$ such that for every $x \in \mathfrak{a}_{1} \cup \mathfrak{a}_{2}, \alpha(x)$ is essentially skew-adjoint, $e^{\overline{\alpha(x)}} \mathcal{D} \subseteq \mathcal{D}$ and

$$
e^{\overline{\alpha(x)}} \alpha(y) e^{-\overline{\alpha(x)}}=\alpha\left(e^{\text {ad } x} y\right) \quad \text { for } \quad y \in \mathfrak{g}^{c} .
$$

Then $\alpha$ integrates to a continuous unitary representation $(\pi, \mathcal{H})$ of $G^{c}$ with $\mathcal{D} \subseteq \mathcal{H}^{\infty}$ and $\alpha(x)=\left.\mathrm{d} \pi(x)\right|_{\mathcal{D}}$ for $x \in \mathfrak{g}^{c}$.

Lemma 5.10. For each $\varphi \in \mathcal{D}^{1}$, the complex linear map

$$
\mathrm{ev}_{\varphi}: \mathfrak{g}_{\mathbb{C}} \rightarrow \mathcal{H}_{K}, \quad x \mapsto \mathcal{L}_{x} \varphi
$$

is continuous. In particular, the representation $\alpha: \mathfrak{g}_{\mathbb{C}} \rightarrow \operatorname{End}(\mathcal{D})$ defined in (5.1) is strongly continuous.

Proof. Since $\mathfrak{g}_{\mathbb{C}}$ and $\mathcal{H}$ are Banach spaces, it suffices to show that the graph of $\left.\mathrm{ev}_{\varphi}\right|_{\mathfrak{g}}$ is closed. This follows from the continuity of the linear functional

$$
\mathfrak{g} \rightarrow \mathbb{C}, \quad x \mapsto\left(\mathcal{L}_{x} \varphi\right)(m)=\mathrm{d} \varphi(m) \beta(x)(m)
$$

which follows from the smoothness of $\varphi$ and our continuity assumption on $\beta$.

Remark 5.11. Let $m \in M$ and $x \in \mathfrak{g}$ be such that $\Phi_{t}^{\beta(x)}(m)$ is defined. We have seen in Lemma 3.4 that

$$
\left.\beta\left(e^{t \operatorname{ad} x} y\right)\right|_{M_{t}}=\left(\Phi_{-t}^{\beta(x)}\right)_{*} \beta(y) \quad \text { for } \quad y \in \mathfrak{g} .
$$

For the corresponding Lie derivatives, this means that

$$
\left(\mathcal{L}_{\beta\left(e^{t \text { ad } x} y\right)} \varphi\right)(m)=\left(\mathcal{L}_{\beta(y)}\left(\varphi \circ \Phi_{-t}^{\beta(x)}\right)\right)\left(\Phi_{t}^{\beta(x)}(m)\right)
$$

for $m \in M_{t}, \varphi \in C^{\infty}(M)$.

Theorem 5.12 (Integrability Theorem). Let $K$ be a smooth positive definite kernel on the manifold $M$ compatible with the smooth right action $(\beta, \sigma)$ of $(\mathfrak{g}, H)$, where $\mathfrak{g}=\mathfrak{h} \oplus \mathfrak{q}$ is a symmetric Banach-Lie algebra and $H$ is a connected Lie group with Lie algebra $\mathfrak{h}$. Let $G^{c}$ be a simply connected Lie group with Lie algebra $\mathfrak{g}^{c}=\mathfrak{h}+i \mathfrak{q}$. Then there exists a unique smooth unitary representation $\left(\pi^{c}, \mathcal{H}_{K}\right)$ such that

(i) $\overline{\mathrm{d} \pi^{c}}(x)=\mathcal{L}_{x}$ for $x \in \mathfrak{h}$.

(ii) $\overline{\mathrm{d} \pi^{c}}(i y)=i \mathcal{L}_{y}$ for $y \in \mathfrak{q}$.

Proof.

Step 1. For $x \in \mathfrak{q}$, we consider the associated selfadjoint operator $\mathcal{L}_{x}$ on $\mathcal{H}_{K}$. Then we obtain a hermitian one-parameter group $e^{t \mathcal{L}_{x}}$ of unbounded selfadjoint operators on $\mathcal{H}_{K}$ defined by a spectral measure $P$ of $\mathcal{L}_{x}$ via

$$
e^{t \mathcal{L}_{x}}:=\int_{\mathbb{R}} e^{t x} d P(x) \quad \text { on } \quad \mathcal{D}\left(e^{t \mathcal{L}_{x}}\right)=\left\{\varphi \in \mathcal{H}^{K}: \int_{\mathbb{R}} e^{2 t x} d P^{\varphi}(x)<\infty\right\},
$$

where $P^{\varphi}(\cdot)=\langle P(\cdot) \varphi, \varphi\rangle$. In particular, we have, for each $t \in \mathbb{R}$, a well-defined domain

$$
\mathcal{D}_{t}=\mathcal{D}\left(e^{t \mathcal{L}_{x}}\right) \subseteq \mathcal{H}_{K}
$$


Let $m \in M_{s}$. Then Theorem 4.6 implies that $K_{m} \in \mathcal{D}_{s}$ with

$$
e^{t \mathcal{L}_{x}} K_{m}=K_{\Phi_{t}^{\beta(x)}(m)} \quad \text { for } \quad 0 \leq t \leq s .
$$

The curve $t \mapsto e^{t \mathcal{L}_{x}} K_{m}$ in $\mathcal{H}_{K}$ extends to a holomorphic function $e^{z \mathcal{L}_{x}} K_{m}$ defined on an open neighborhood of the strip $0 \leq \operatorname{Re} z \leq s$. Accordingly, for $y \in \mathfrak{g}$, the function

$$
t \mapsto\left\langle e^{t \mathcal{L}_{x}} K_{m}, \mathcal{L}_{y} K_{n}\right\rangle
$$

extends to a holomorphic function

$$
z \mapsto\left\langle e^{z \mathcal{L}_{x}} K_{m}, \mathcal{L}_{y} K_{n}\right\rangle .
$$

For $x \in \mathfrak{q}, y \in \mathfrak{g}$ and $m, n \in M_{s}$ we get:

$$
\begin{aligned}
& \left\langle e^{t \mathcal{L}_{x}} K_{m}, \mathcal{L}_{\tau(y)} K_{n}\right\rangle=\left\langle K_{\Phi_{t}^{\beta(x)}(m)}, \mathcal{L}_{\tau(y)} K_{n}\right\rangle=\overline{\left(\mathcal{L}_{\tau(y)} K_{n}\right)\left(\Phi_{t}^{\beta(x)}(m)\right)} \\
& =\overline{\left(\mathcal{L}_{e^{t \text { ad } x} \tau(y)}\left(K_{n} \circ \Phi_{t}^{\beta(x)}\right)\right)(m)} \quad \text { by } \\
& =\left\langle\mathcal{L}_{e^{t \text { ad } x} \tau(y)}^{*} K_{m}, e^{t \mathcal{L}_{x}} K_{n}\right\rangle \\
& =-\left\langle\mathcal{L}_{e^{-t \operatorname{ad} x} y} K_{m}, e^{t \mathcal{L}_{x}} K_{n}\right\rangle .
\end{aligned}
$$

By analytic extension (cf. [KL81, Lemma 2]), we now arrive with Lemma 5.10] at the relation

$$
\left\langle e^{z \mathcal{L}_{x}} K_{m}, \mathcal{L}_{\tau(y)} K_{n}\right\rangle=-\left\langle\mathcal{L}_{e^{-z \text { ad } x} y} K_{m}, e^{\bar{z} \mathcal{L}_{x}} K_{n}\right\rangle \quad \text { for } \quad 0 \leq \operatorname{Re} z \leq s
$$

and we get in particular

$$
\left\langle e^{i \mathcal{L}_{x}} K_{m}, \mathcal{L}_{\tau(y)} K_{n}\right\rangle=-\left\langle\mathcal{L}_{e^{-i \text { ad } x} y} K_{m}, e^{-i \mathcal{L}_{x}} K_{n}\right\rangle .
$$

This relation leads to $e^{i \mathcal{L}_{x}} K_{m} \in \mathcal{D}_{y}$ with

$$
\mathcal{L}_{y} e^{i \mathcal{L}_{x}} K_{m}=e^{i \mathcal{L}_{x}} \mathcal{L}_{e^{-i \operatorname{ad} x} y} K_{m} \quad \text { for } \quad m \in M_{s}, y \in \mathfrak{g}_{\mathbb{C}} .
$$

From $M=\bigcup_{s>0} M_{s}$ we thus derive $e^{i \mathcal{L}_{x}} \mathcal{H}_{K}^{0} \subseteq \mathcal{D}^{1}$ with

$$
\left.\mathcal{L}_{y} e^{i \mathcal{L}_{x}}\right|_{\mathcal{H}_{K}^{0}}=\left.e^{i \mathcal{L}_{x}} \mathcal{L}_{e^{-i \text { ad } x} y}\right|_{\mathcal{H}_{K}^{0}},
$$

resp.,

$$
\left.e^{-i \mathcal{L}_{x}} \mathcal{L}_{y} e^{i \mathcal{L}_{x}}\right|_{\mathcal{H}_{K}^{0}}=\left.\mathcal{L}_{e^{-i \text { ad } x} y}\right|_{\mathcal{H}_{K}^{0}} \quad \text { for } \quad x \in \mathfrak{q}, y \in \mathfrak{g}_{\mathbb{C}}
$$

Step 2. It follows from Step 1 that, for $m, n \in M, x \in \mathfrak{q}$ and $y \in \mathfrak{g}^{c}$, we have

$$
\left\langle e^{i \mathcal{L}_{x}} K_{m}, \mathcal{L}_{y} K_{n}\right\rangle=\left\langle K_{m}, \mathcal{L}_{e^{-i \text { ad } x} y} e^{-i \mathcal{L}_{x}} K_{n}\right\rangle
$$

and by continuity

$$
\left\langle e^{i \mathcal{L}_{x}} \varphi, \mathcal{L}_{y} K_{n}\right\rangle=\left\langle\varphi, \mathcal{L}_{e^{-i \text { ad } x} y} e^{-i \mathcal{L}_{x}} K_{n}\right\rangle=-\left\langle\mathcal{L}_{e^{-i \text { ad } x} y} \varphi, e^{-i \mathcal{L}_{x}} K_{n}\right\rangle
$$

for $\varphi \in \mathcal{D}_{e^{-i \text { ad } x} y}$. Hence $e^{i \mathcal{L}_{x}} \varphi \in \mathcal{D}\left(\left(\left.\mathcal{L}_{y}\right|_{K_{n}}\right)^{*}\right)$. If $y \in \mathfrak{h} \cup i \mathfrak{q}$, then by Lemma 5.8 and Corollary $\left[5.7\right.$ we have $\left(\left.\mathcal{L}_{y}\right|_{\mathcal{H}_{K}^{0}}\right)^{*}=-\mathcal{L}_{y}$. It follows that

$$
e^{i \mathcal{L}_{x}} \mathcal{D}^{1} \subseteq \mathcal{D}^{1} \quad \text { and }\left.\quad e^{-i \mathcal{L}_{x}} \mathcal{L}_{y} e^{i \mathcal{L}_{x}}\right|_{\mathcal{D}^{1}}=\left.\mathcal{L}_{e^{-i \text { ad } x} y}\right|_{\mathcal{D}^{1}} \quad \text { for } \quad x \in \mathfrak{q}, y \in \mathfrak{g}^{c}
$$

and the commutation relation implies $e^{i \mathcal{L}_{x}} \mathcal{D} \subseteq \mathcal{D}$. 
Step 3. For $x \in \mathfrak{h}$ we have

$$
e^{\mathcal{L}_{x}} \varphi=\pi^{H}(\exp x) \varphi=\varphi \circ \sigma_{\exp x} \quad \text { for } \quad \varphi \in \mathcal{H}_{K} .
$$

Therefore the relation

$$
e^{-\mathcal{L}_{x}} \mathcal{L}_{y} e^{\mathcal{L}_{x}}=\mathcal{L}_{e^{- \text {ad } x} y} \quad \text { for } \quad x \in \mathfrak{h}, y \in \mathfrak{g}^{c}
$$

follows from

$$
\left(\sigma_{\exp x}\right)_{*} \beta(y)=\beta\left(e^{-\operatorname{ad} x} y\right) \quad \text { for } \quad x \in \mathfrak{h}, y \in \mathfrak{g}
$$

(Remark 5.5). Since $\mathcal{D}$ is dense by Proposition 5.6, we can now apply Theorem 5.9 to complete the proof.

Remark 5.13. Note that (i) implies that the restriction of $\pi^{c}$ to the integral subgroup $\langle\exp \mathfrak{h}\rangle \subseteq G^{c}$ induces the same representation as $\pi^{H}$ on the universal covering group $\widetilde{H}$ of $H$.

5.3. Examples. We now discuss a series of examples illustrating various situations in which the Integrability Theorem 5.12 applies.

Example 5.14. (a) Let $(G, \tau)$ be a symmetric Banach-Lie group with Lie algebra $\mathfrak{g}=\mathfrak{h}+\mathfrak{q}, H \subseteq G$ the integral subgroup corresponding to the closed Lie subalgebra $\mathfrak{h}$, and $U=U H \subseteq G$ be an open subset. We write $g^{\sharp}=\tau(g)^{-1}$ for $g \in G$. Then a function $\varphi: U U^{\sharp} \rightarrow \mathbb{C}$ is called $\tau$-positive definite if the kernel

$$
K(x, y):=\varphi\left(x y^{\sharp}\right)
$$

is positive definite.

Then $\sigma_{h}(g):=g h$ and $\beta(x)(g):=g . x$ defines a smooth right action of $(\mathfrak{g}, H)$ on $U$ and the kernel $K$ is compatible with $(\beta, \sigma)$. Therefore we obtain for each simply connected Lie group $G^{c}$ a corresponding unitary representation $\pi^{c}$ on $\mathcal{H}_{K} \subseteq$ $C^{\infty}(U, \mathbb{C})$ with

and

$$
\left(\pi^{c}(h) \psi\right)(g)=\psi(g h) \quad \text { for } \quad g \in U, h \in H
$$

$$
\mathrm{d} \pi^{c}(i y) \psi=i \mathcal{L}_{y} \psi \quad \text { for } \quad \psi \in \mathcal{H}_{K}^{\infty}, y \in \mathfrak{q} .
$$

(b) If $\rho: G \rightarrow \mathrm{GL}(V)$ is a smooth action of the Banach-Lie group $G$ on the locally convex space and $(\cdot, \cdot)$ is a positive definite scalar product on $V$ satisfying

$$
(\rho(g) v, w)=\left(v, \rho\left(g^{\sharp}\right) w\right) \quad \text { for } \quad g \in G, v, w \in V,
$$

then $\rho$ defines in particular a smooth right action $(\beta, \sigma)$ on the manifold $M:=V$ by

$$
\sigma_{h}=\rho(h)^{-1} \quad \text { and } \quad \beta(x) v=-\mathrm{d} \rho(x) v,
$$

and the kernel $K(v, w):=(w, v)$ is compatible. Therefore we obtain for each simply connected Lie group $G^{c}$ a smooth unitary representation on $\mathcal{H}_{K}$, which can be identified with the Hilbert completion of $V \subseteq \mathcal{H}_{K}^{\infty}$, and $\left.\pi(h)\right|_{V}=\rho(h)$ for $h \in H$.

This situation occurs in particular for finite dimensional representations $(\rho, V)$ of real reductive Lie groups. In this case $\tau$ is a Cartan involution on $G$, a scalar product as above always exists, and the group $G^{c}$ is compact. Here the passage between unitary representations of $G^{c}$ and representations of $G$ is Weyl's Unitary Trick, used by H. Weyl to study representations of $G$ by means of unitary representations of $G^{c}$.

\footnotetext{
${ }^{2}$ Here $G \times T(G) \rightarrow T(G),(g, v) \mapsto g \cdot v=T\left(\lambda_{g}\right) v$ is the canonical left action of $G$ on the tangent bundle $T(G)$, so that $\beta(x)(g)=g \cdot x$ is the left invariant vector field with $\beta(x)(\mathbf{1})=x$.
} 
Example 5.15. Let $V$ be a Banach space and $\Omega \subseteq V$ be an open domain. Further, let $\mu$ be a positive measure on the smallest $\sigma$-algebra in the topological dual space $V^{\prime}$ for which all evaluation maps $\operatorname{ev}_{v}(\alpha):=\alpha(v)$ are measurable. We assume that the Laplace transform

$$
\mathcal{L}(\mu)(x):=\int_{V^{\prime}} e^{-\alpha(x)} d \mu(\alpha)
$$

defines a smooth function on $\Omega$. This is always the case if $\operatorname{dim} V<\infty$ and $\mathcal{L}(\mu)(x)<$ $\infty$ for $x \in \Omega$ (cf. [Ne0, Prop. V.4.6]). Then

$$
K(x, y):=\mathcal{L}(\mu)\left(\frac{x+y}{2}\right)
$$

defines a smooth positive definite kernel on $\Omega$.

We consider the symmetric Lie group $(G, \tau)=\left(V,-\mathrm{id}_{V}\right)$ for which $H=\{0\}$ and $\mathfrak{g}=\mathfrak{q}=V$. For every $v \in V$, the corresponding constant vector field $\beta(v)(x)=v$ is locally integrable with maximal local flow given by $\Phi(t, x)=x+t v$ whenever $x+t v \in \Omega$. We thus obtain a smooth right action of $(\mathfrak{g}, H)$ on $\Omega$. Since the kernel depends only on $x+y$, the vector fields $\beta(v)$ are $K$-symmetric. Therefore Theorem 5.12 guarantees the existence of a corresponding unitary representation of the Banach-Lie group $G^{c}=i V$ on the corresponding reproducing kernel Hilbert space $\mathcal{H}_{K}$.

As the kernel $K$ extends holomorphically to the tube domain $T_{\Omega}=\Omega+i V$ by

$$
K(z, w):=\mathcal{L}(\mu)\left(\frac{z+\bar{w}}{2}\right),
$$

the corresponding Hilbert space can be identified with a space of holomorphic functions on $T_{\Omega}$ and the unitary representation of $G^{c}=i V$ is simply given by $\left(\pi^{c}(i v) f\right)(z):=f(z+i v)$.

One can show that $\mathcal{H}_{K} \cong L^{2}\left(V^{\prime}, \mu\right)$ and that the elements of $\mathfrak{g}=V$ act naturally by multiplication operators on $L^{2}\left(V^{\prime}, \mu\right)$. In particular, the operator $\mathcal{L}_{v}^{K}$ is unbounded from above and below if the support of the measure $\left(\operatorname{ev}_{v}\right)_{*} \mu$ on $\mathbb{R}$ has this property.

Example 5.16. (a) Let $\mathcal{A}$ be a real Banach-*-algebra and $G:=\mathcal{A}^{\times}$be its group of units which is a Banach-Lie group whose Lie algebra $\mathfrak{g}$ is $(\mathcal{A},[\cdot, \cdot])$. If $\mathcal{A}$ is not unitial, we embed it into $\mathcal{A}_{1}:=\mathbb{R} \mathbf{1} \oplus \mathcal{A}$ and define $\mathcal{A}^{\times}:=(\mathbf{1}+\mathcal{A}) \cap \mathcal{A}_{1}^{\times}$. Then $G$ is a symmetric Banach-Lie group with respect to $\tau(a):=\left(a^{*}\right)^{-1}$ and $H=\left\{a \in \mathcal{A}^{\times}: a^{*}=a^{-1}\right\}$ is the unitary/orthogonal group of $\mathcal{A}$. If $\mathcal{A}_{\mathbb{C}}$ is the complexification of $\mathcal{A}$ and $(x+i y)^{*}:=x^{*}-i y^{*}$ is the antilinear extension of $*$ to $\mathcal{A}_{\mathbb{C}}$, then the unitary group $\mathrm{U}\left(\mathcal{A}_{\mathbb{C}}\right)$ is a Banach-Lie group with Lie algebra

$$
\mathfrak{g}^{c}=\left\{a \in \mathcal{A}: a^{*}=-a\right\}+i\left\{b \in \mathcal{A}: b^{*}=b\right\}=\left\{c \in \mathcal{A}_{\mathbb{C}}: c^{*}=-c\right\}=\mathfrak{u}\left(\mathcal{A}_{\mathbb{C}}\right) .
$$

Accordingly, we write $G^{c}$ for the simply connected covering group of the identity component $\mathrm{U}(\mathcal{A})_{0}$.

Let $S:=\{a \in \mathcal{A}:\|a\|<1\}$ be the open unit ball in $\mathcal{A}$ and observe that this is a $*$-semigroup. Let $\pi_{S}: S \rightarrow B(\mathcal{H})$ be a $*$-representation of $S$ and $v \in \mathcal{H}$ be a smooth vector for this representation, so that we obtain the positive definite smooth function $\varphi(s):=\langle\pi(s) v, v\rangle$ and the corresponding smooth kernel $K(s, u):=\varphi\left(s u^{*}\right)$ on $S \times S$.

Restricting the right multiplication action of $G=\mathcal{A}^{\times}$to $S$, we obtain a smooth right action of $(\mathfrak{g}, H)$, for which the vector fields are given by $\beta(x) a=a x$. We then 
have

$$
\left(\mathcal{L}_{x}^{1} K\right)(s, u)=\left.\frac{d}{d t}\right|_{t=0} \varphi\left(s e^{t x} u^{*}\right)=\left.\frac{d}{d t}\right|_{t=0} \varphi\left(s\left(u e^{t x^{*}}\right)\right)=\left(\mathcal{L}_{x^{*}}^{2} K\right)(s, u),
$$

so that the kernel $K$ is compatible with the smooth right action.

We thus obtain from Theorem 5.12 a smooth unitary representation of $G^{c}$ on the reproducing kernel space $\mathcal{H}_{\varphi} \subseteq C^{\infty}(S)$ which can be identified with the cyclic subspace $\mathcal{H}_{v}:=\overline{\operatorname{span} \pi(S) v} \subseteq \mathcal{H}$.

(b) If $\mathcal{A}=M_{n}(\mathbb{R})$ with $A^{*}=A^{\top}$ and $s \in \mathbb{R}$ is such that the kernel

$$
K(x, y):=\operatorname{det}\left(\mathbf{1}-x y^{\top}\right)^{-s}=\operatorname{det}\left(\mathbf{1}-x y^{*}\right)^{-s}
$$

on the open unit ball $S$ is positive definite, then we likewise obtain a unitary representation of the group $G^{c}=\widetilde{\mathrm{U}}_{n}(\mathbb{C})$ on the corresponding reproducing kernel space.

In this case the extension can also be obtained from the observation that the positive definiteness of the kernel $K$ implies the positive definiteness of the kernel

$$
K_{\mathbb{C}}(x, y):=\operatorname{det}\left(\mathbf{1}-x y^{*}\right)^{-s}
$$

on the open unit ball $S_{\mathbb{C}} \subseteq M_{n}(\mathbb{C})$ ([NO14, Thm. A.1]).

Example 5.17 (Application to operator-valued kernels). (a) Let $(G, \tau)$ be a symmetric Banach-Lie group and write $g^{\sharp}=\tau(g)^{-1}$. We consider a smooth right action of $G$ on the manifold $X$, a complex Hilbert space $V$, and a kernel $Q: X \times X \rightarrow B(V)$. We assume that we have a function $J: G \times X \rightarrow \mathrm{GL}(V),(g, x) \mapsto J_{g}(x)$ satisfying the cocycle condition

$$
J_{g_{1} g_{2}}(x)=J_{g_{1}}(x) J_{g_{2}}\left(x . g_{1}\right) \quad \text { for } \quad g_{1}, g_{2} \in G, x \in X,
$$

and that the kernel $Q$ satisfies the corresponding invariance relation

$$
J_{g}(x) Q(x . g, y)=Q\left(x, y \cdot g^{\sharp}\right) J_{g^{\sharp}}(y)^{*} \quad \text { for } \quad x, y \in X, g \in G
$$

(cf. [Ne00, Prop. II.4.3]). On the set $M:=X \times V$, we then obtain a $G$-right action by

$$
(x, v) . g:=\left(x . g, J_{g}(x)^{*} v\right) .
$$

We also obtain a positive definite kernel

$$
K: M \times M \rightarrow \mathbb{C}, \quad K((x, v),(y, w)):=\langle Q(x, y) w, v\rangle
$$

which satisfies the natural covariance condition

$$
\begin{aligned}
K((x, v) . g,(y, w)) & =K\left(\left(x \cdot g, J_{g}(x)^{*} v\right),(y, w)\right)=\left\langle Q(x \cdot g, y) w, J_{g}(x)^{*} v\right\rangle \\
& =\left\langle J_{g}(x) Q(x \cdot g, y) w, v\right\rangle=\left\langle Q\left(x, y \cdot g^{\sharp}\right) J_{g^{\sharp}}(y)^{*} w, v\right\rangle \\
& =K\left((x, v),\left(y \cdot g^{\sharp}, J_{g^{\sharp}}(y)^{*} w\right)\right)=K\left((x, v),(y, w) \cdot g^{\sharp}\right) .
\end{aligned}
$$

Let $X_{+} \subseteq X$ be an open $H$-invariant subset on which the kernel $Q$ is positive definite, so that $K$ is positive definite on $M_{+}:=X_{+} \times V$. The corresponding reproducing kernel Hilbert space $\mathcal{H}_{K} \subseteq \mathbb{C}^{M_{+}}$consists of functions that are continuous and antilinear in the second argument, and it is easy to see that the map

$$
\Gamma: \mathcal{H}_{Q} \rightarrow \mathcal{H}_{K}, \quad \Gamma(f)(x, v):=\langle f(x), v\rangle
$$

is unitary. For the $G$-action $(g . f)(x):=J_{g}(x) f(x . g)$ on $V^{X}$, we have

$$
\Gamma(g . f)(x, v)=\left\langle J_{g}(x) f(x . g), v\right\rangle=\left\langle f(x . g), J_{g}(x)^{*} v\right\rangle=\Gamma(f)((x, v) . g),
$$


so that $\Gamma$ intertwines it with the action on $\mathbb{C}^{M}$ by

$$
(g . F)(x, v):=F((x, v) \cdot g) .
$$

Let us assume that the $G$-action on $M_{+}$is smooth, i.e., that $G \times X_{+} \times V \rightarrow$ $V,(g, x, v) \mapsto J_{g}(x)^{*} v$ is smooth. Then we obtain a smooth right action of $(\mathfrak{g}, H)$ on $M_{+}$compatible with the kernel $K$, and thus Theorem 5.12 yields a unitary representation of $G^{c}$ on the Hilbert $\mathcal{H}_{K} \cong \mathcal{H}_{Q}$.

(b) A concrete example of this type is obtained from the kernel $K(x, y):=e^{\langle x, y\rangle}$ on the infinite dimensional real Hilbert space $\mathcal{H}$. Then $G:=\mathrm{GL}(\mathcal{H})$ is a Banach-Lie group and $\tau(g):=\left(g^{\top}\right)^{-1}$ is an involutive automorphism satisfying

$$
K(g . x, y)=K\left(x, g^{\top} . y\right) \quad \text { for } \quad g \in G, x, y \in \mathcal{H} .
$$

As the unitary group $\mathrm{U}\left(\mathcal{H}_{\mathbb{C}}\right)$ is simply connected by Kuiper's Theorem, Theorem 5.12 yields a unitary representation of $\mathrm{U}\left(\mathcal{H}_{\mathbb{C}}\right)$ on the Fock space $\mathcal{H}_{K} \subseteq \mathbb{C}^{\mathcal{H}}$ (which can also be obtained more directly by analytic extension of the kernel).

5.4. A generalization of the Lüscher-Mack Theorem. In this subsection we prove a generalization of the Lüscher-Mack Theorem which applies to a class of semigroups that need not have a polar decomposition.

Theorem 5.18 (Generalized Lüscher-Mack Theorem). Let $(G, \tau)$ be a connected symmetric Banach-Lie group. For $H:=\left(G^{\tau}\right)_{0}$, we consider an open subsemigroup $S \subseteq G$ with $S H=S$, and which is invariant under the involution $s \mapsto s^{\sharp}:=\tau(s)^{-1}$. Then, for every non-degenerate strongly continuous smooth representation 3 of the involutive semigroup $(S, \sharp)$, the following assertions hold:

(a) There exist a smooth unitary representation $\pi^{H}$ of $H$ in $\mathcal{H}$ and a Lie algebra representation $\alpha: \mathfrak{g}_{\mathbb{C}} \rightarrow \operatorname{End}(\mathcal{D})$ on the dense domain $\mathcal{D} \subseteq \mathcal{H}$ such that $\alpha(x)$ is essentially skew-adjoint for $x \in \mathfrak{h} \cup i \mathfrak{q}$ and

(i) $\pi(s h)=\pi(s) \pi^{H}(h)$ for $s \in S$ and $h \in H$.

(ii) If $x \in \mathfrak{q}$ and $\exp t x \in S$ for $t>0$, then $\pi(\exp t x)=e^{\overline{t \alpha(x)}}$.

(iii) $\mathcal{D}$ consists of smooth vectors for $\pi^{H}$ and $\alpha(x) v=\mathrm{d} \pi^{H}(x) v$ for $v \in \mathcal{D}$.

(b) The representation $\left.\alpha\right|_{\mathfrak{g}^{c}}$ integrates to a (smooth) unitary representation of any simply connected Lie group $G^{c}$ with Lie algebra $\mathfrak{g}^{c}$.

Proof. First we note that every non-degenerate smooth representation $(\pi, \mathcal{H})$ of $(S, \sharp)$ is a direct sum of cyclic ones generated by a smooth vector (see $\mathrm{Ne00}$, Lemma II.4.2(iv)]).

If the representation is generated by the smooth vector $v$, then the kernel $K(s, t)$ $:=\left\langle\pi\left(s t^{\sharp}\right) v, v\right\rangle$ on $S \times S$ is smooth and positive definite and satisfies

$$
K(s u, t)=K\left(s, t u^{\sharp}\right) \quad \text { for } \quad s, t, u \in S .
$$

Moreover, the unitary isomorphism

$$
\Gamma: \mathcal{H} \rightarrow \mathcal{H}_{K} \subseteq C^{\infty}(S, \mathbb{C}), \quad \Gamma(w)(s):=\langle\pi(s) w, v\rangle
$$

is an intertwining operator, where the representation $\mathcal{H}_{K}$ is given by

$$
\left(\pi_{K}(s) f\right)(t):=f(t s) .
$$

Hence the assertion follows from Example 5.14(a).

\footnotetext{
${ }^{3}$ Smoothness of a representation means that the linear subspace $\mathcal{H}^{\infty}$ of all vectors with smooth orbit map is dense in $\mathcal{H}$.
} 
Remark 5.19. If $G$ is finite dimensional, then every non-degenerate continuous representation of $S$ is smooth. In fact, for every $\varphi \in C_{c}^{\infty}(S)$, the image of the operators defined by

$$
\pi(\varphi) v=\int_{S} \varphi(s) \pi(s) v d \mu_{G}(s)
$$

are smooth vectors. Here $\mu_{G}$ denotes a left Haar measure on $G$.

\section{LOCAL REPRESENTATIONS}

In this section we give two proofs of P.E.T. Jorgensen's theorem on the analytic continuation of local representations. The first one only applies to finite dimensional groups. As Jorgensen's original proof, its first part relies on Simon's Exponentiation Theorem Si72. However, we are able, using analytic continuation, to simplify substantially its second part. As a result, axioms (L5) and (L6) from [Jo86] are not needed anymore. The second proof requires globality of the representation on the subgroup $H$ and a smoothness condition but has the advantage of applying to infinite dimensional Lie groups as well. The latter version can be derived from Theorem 5.12

\subsection{Local representations of finite dimensional groups.}

Definition 6.1. Let $(G, H, \tau)$ be a symmetric Lie group and $\mathfrak{h}=\operatorname{ker}(\tau-\mathbf{1})$, $\mathfrak{q}=\operatorname{ker}(\tau+\mathbf{1})$ as above. Also let $g^{\sharp}:=\tau(g)^{-1}$. Let $\mathcal{H}$ be a Hilbert space and $U \subseteq G$ be an identity neighborhood. Assume that, for every $g \in U$, we are given a densely defined operator $\pi(g): \mathcal{D}(\pi(g)) \rightarrow \mathcal{H}$ on $\mathcal{H}$ and that there exists a continuous unitary representation $\left(\pi_{H}, \mathcal{H}\right)$ of $H$ such that $\left.\pi_{H}(h)\right|_{\mathcal{D}(\pi(h))}=\pi(h)$ for every $h \in U \cap H$. Let $\mathcal{D}$ be a dense subspace in $\mathcal{H}$. Then $(\pi, \mathcal{D}, \mathcal{H})$ is called a local representation of $(G, H, \tau)$ if the following assumptions are satisfied:

(L0) If $g, g^{\sharp} \in U$, then $\pi\left(g^{\sharp}\right) \subseteq \pi(g)^{*}$.

$(\mathrm{L} 1) \mathcal{D} \subseteq \mathcal{D}(\pi(g))$ for every $g \in U$.

(L2) If $g_{1}, g_{2}$ and $g_{1} g_{2}$ are in $U$, then

$$
\pi\left(g_{2}\right) \mathcal{D} \subseteq \mathcal{D}\left(\pi\left(g_{1}\right)\right) \quad \text { and }\left.\quad \pi\left(g_{1} g_{2}\right)\right|_{\mathcal{D}}=\left.\pi\left(g_{1}\right) \pi\left(g_{2}\right)\right|_{\mathcal{D}}
$$

(L3) There exists a starlike 0-neighborhood $U_{\mathfrak{q}}$ in $\mathfrak{q}$ such that $\exp U_{\mathfrak{q}} \subseteq U$ and for every $v \in \mathcal{D}$

$$
\lim _{t \rightarrow 0} \pi(\exp t y) v=v .
$$

It follows from (L1-3) and [Fro80, Theorem I.1] that, for $y \in \mathfrak{q}$, the local semigroup $\pi(\exp (t y))$ has a unique extension as a local selfadjoint semigroup $e^{t B_{y}}$, where $B_{y}$ is a selfadjoint operator with $\mathcal{D} \subseteq \mathcal{D}\left(B_{y}\right)$. We will moreover assume:

(L4) If $y \in \mathfrak{q}$, then $B_{y} \mathcal{D} \subseteq \mathcal{D}$.

For $x \in \mathfrak{h}$, we write $B_{x}$ for the skew-adjoint generator of $\pi_{H}(\exp t x)$.

Theorem 6.2. Let $(\pi, \mathcal{D}, \mathcal{H})$ be a local representation of the finite dimensional Lie group $G$. Let $\mathfrak{g}^{c}=\mathfrak{h}+i \mathfrak{q}$ be the c-dual Lie algebra and let $G^{c}$ be the corresponding simply connected Lie group. Then there exists a unitary representation $\left(\pi^{c}, \mathcal{H}\right)$ of $G^{c}$ such that

(i) $\overline{\mathrm{d} \pi^{c}(x)}=B_{x}$ for $x \in \mathfrak{h}$,

(ii) $\overline{\mathrm{d} \pi^{c}(i y)}=i B_{y}$ for $y \in \mathfrak{q}$. 
Proof.

Step 1. The first step of the proof follows [Jo86, Observation 1-3]. For $x \in \mathfrak{h}_{1}:=$ $[\mathfrak{q}, \mathfrak{q}]$ one shows that $\mathcal{D} \subseteq \mathcal{D}\left(B_{x}\right)$ and $B_{x} \mathcal{D} \subseteq \mathcal{D}$. It follows that one can define a representation $\alpha$ of $\mathfrak{g}_{1}^{c}:=\mathfrak{h}_{1}+i \mathfrak{q}$ on $\mathcal{D}$ by skew-symmetric operators via the formula

$$
\alpha(x+i y):=\left.B_{x}\right|_{\mathcal{D}}+\left.i B_{y}\right|_{\mathcal{D}} .
$$

Assumption (L4) implies with [Jo87, Lemma A] that $\mathcal{D}$ consists of analytic vectors for every $y \in \mathfrak{q}$. Since, by construction, the Lie algebra $\mathfrak{g}_{1}^{c}$ is generated by $\mathfrak{q}$, Simon's Exponentiation Theorem Si72] applies and $\alpha$ integrates to a unitary representation $\left(\pi_{1}^{c}, \mathcal{H}\right)$ of the simply connected group $G_{1}^{c}$ with Lie algebra $\mathfrak{g}_{1}^{c}$.

Step 2. We consider the semidirect product $H \ltimes G_{1}^{c}$ where the action of $H$ on $G_{1}^{c}$ is obtained by integrating the adjoint action. We claim that the unitary representations $\pi_{H}$ and $\pi_{1}^{c}$ combine to a unitary representation $\widehat{\pi}$ of $H \ltimes G_{1}^{c}$. It suffices to show that for $h \in H$ and $y \in \mathfrak{q}$ the commutation relation

$$
\pi_{H}(h) \pi_{1}^{c}(\exp i y)=\pi_{1}^{c}(\exp i \operatorname{Ad}(h) y) \pi_{H}(h)
$$

holds. Let $s>0$ such that $s y$ and $s \operatorname{Ad}(h) y$ are in $U_{\mathfrak{q}}$. Then, for $\operatorname{Re}(z)<s$ and $v, w \in \mathcal{D}$, the maps $F(z)=\left\langle\pi(h) e^{z B_{y}} v, w\right\rangle$ and $G(z)=\left\langle e^{z B_{\mathrm{Ad}(h) y}} \pi(h) v, w\right\rangle$ are analytic. Since $F(t)=G(t)$ for $t \in(0, s)$ by (L2), we have by analytic continuation $F(i)=G(i)$ and (6.1) follows. The kernel of the representation $\widehat{\pi}$ contains the subgroup $\Delta:=\left\{\left(h, h^{-1}\right): h \in h\right\} \subseteq H \ltimes G_{1}^{c}$, so that it factors through a representation $\pi^{c}$ of $G^{c} \cong\left(H \ltimes G_{1}^{c}\right) / \Delta$.

6.2. Local representations of infinite dimensional groups. Since a (continuous) unitary representation of an infinite dimensional Lie group does not necessarily possesses smooth vectors ( $[\mathrm{Ne10}]$ ), we need to add the following assumption in the definition of a local representation for an infinite dimensional Lie group.

(LI1) There exists a possibly finer locally convex topology on $\mathcal{D}$ such that the action map $U \times \mathcal{D} \rightarrow \mathcal{H},(g, v) \mapsto \pi(g) v$, is smooth.

We then obtain an unbounded operator $\mathrm{d} \pi(x): \mathcal{D} \rightarrow \mathcal{H}$ by the formula

$$
\mathrm{d} \pi(x) v:=\left.\frac{\mathrm{d}}{\mathrm{d} t}\right|_{t=0} \pi(\exp t x) v, v \in \mathcal{D} .
$$

To be able to use the results of Sections 3-4, we also add the following assumption:

(LI2) $U H=U$.

It turns out that with these new assumptions, condition (L4) is not needed anymore.

Theorem 6.3. Let $(\pi, \mathcal{D}, \mathcal{H})$ be a local representation of the Banach-Lie group $G$ satisfying (L1-3) and (LI1/2). Let $\mathfrak{g}^{c}=\mathfrak{h}+i \mathfrak{q}$ be the $c$-dual Lie algebra and $G^{c}$ be a simply connected Lie group with Lie algebra $G^{c}$. Then there exists a unitary representation $\left(\pi^{c}, \mathcal{H}\right)$ of $G^{c}$ such that

$$
\left.\mathrm{d} \pi^{c}(x+i y)\right|_{\mathcal{D}}=\mathrm{d} \pi(x)+i \mathrm{~d} \pi(y) \quad \text { for } \quad x \in \mathfrak{h}, y \in \mathfrak{q} .
$$

Proof. Let us define $\gamma(g, v):=\pi\left(g^{\sharp}\right) v$ where $g^{\sharp}:=\tau(g)^{-1}$. Then

$$
K((g, v),(k, w))):=\langle\gamma(k, w), \gamma(g, v)\rangle
$$

is a smooth positive definite kernel on $U \times \mathcal{D}$ and $\Gamma: \mathcal{H} \rightarrow \mathcal{H}_{K} \subseteq C^{\infty}(U \times \mathcal{D}, \mathbb{C})$,

$$
\Gamma(w):=\langle w, \gamma(g, v)\rangle
$$


is a Hilbert space isomorphism. Let $(\beta, \sigma)$ be the smooth right action of $(\mathfrak{g}, H)$ on $U \times \mathcal{D}$ defined by

$$
\beta(x)(g, v):=(g . x, 0), \quad \sigma(h)(g, v)=(g h, v), \quad x \in \mathfrak{g}, h \in H, g \in U, v \in \mathcal{D} .
$$

For $h \in H$ we have $h^{\sharp}=h^{-1}$ and we thus get

$$
K((g, v) h,(k, w))=K\left((g, v),(k, w) h^{-1}\right) .
$$

If $x \in \mathfrak{g}$, then $\Phi_{t}^{\beta(x)}(g, v)=(g \exp t x, v)$ and

$$
\begin{aligned}
\mathcal{L}_{\beta(x)}^{1} K((g, v),(k, w)) & =\left.\frac{\mathrm{d}}{\mathrm{d} t}\right|_{t=0}\left\langle\pi\left(k^{\sharp}\right) w, \pi\left((g \exp t x)^{\sharp}\right) v\right\rangle \\
& =\left.\frac{\mathrm{d}}{\mathrm{d} t}\right|_{t=0}\left\langle\pi\left((k \exp -t \tau(x))^{\sharp}\right) w, \pi\left(g^{\sharp}\right) v\right\rangle \\
& =-\mathcal{L}_{\beta(\tau(x))}^{2} K((g, v),(k, w)) .
\end{aligned}
$$

Hence $K$ is $\beta$-compatible and by Theorem $5.12, \mathcal{H}_{K}$ carries on

$$
\mathcal{D}_{K}:=\left\{\varphi \in \mathcal{H}_{K}:\left(\forall n \in \mathbb{N}_{0}, x_{1}, \ldots, x_{n} \in \mathfrak{g}\right) \mathcal{L}_{\beta\left(x_{n}\right)} \ldots \mathcal{L}_{\beta\left(x_{1}\right)} \varphi \in \mathcal{H}_{K}\right\}
$$

a representation $\alpha$ of $\mathfrak{g}_{\mathbb{C}}$ such that $\left.\alpha\right|_{\mathfrak{g}^{c}}$ integrates to a unitary representation of $G^{c}$. To complete the proof, it remains to observe that $\mathcal{D} \subseteq \Gamma^{-1}\left(\mathcal{D}_{K}\right)$ and $\Gamma(\mathrm{d} \pi(x) w)=$ $\mathcal{L}_{\beta(x)} \Gamma(w)$ for $x \in \mathfrak{g}$.

\section{Generalization to Distribution Kernels}

In this section we consider the case where $M$ is a finite dimensional manifold and $K \in C^{-\infty}(M \times M)$ is a positive definite distribution compatible with a smooth right action $(\beta, \sigma)$ of $(\mathfrak{g}, H)$. Our main integrability theorem is Theorem 7.9 .

\subsection{Geometric Fröhlich Theorem for distributions.}

Definition 7.1. Let $M$ be a finite dimensional smooth manifold and let $K \in$ $C^{-\infty}(M \times M)$ be a distribution, i.e., an antilinear continuous functional on the LF space $C_{c}^{\infty}(M \times M) \rightarrow \mathbb{C}$. We say that $K$ is positive definite if

$$
\langle\varphi, \psi\rangle_{K}:=K(\bar{\varphi} \otimes \psi)
$$

defines a positive semidefinite hermitian form on $C_{c}^{\infty}(M)$.

If $K$ is positive definite, then we write $\mathcal{H}_{K}$ for the corresponding Hilbert space completion and $K_{\varphi}$ for the image of $\varphi \in C_{c}^{\infty}(M)$ in $\mathcal{H}_{K}$. Since the canonical map $\iota: C_{c}^{\infty}(M) \rightarrow \mathcal{H}_{K}, \varphi \mapsto K_{\varphi}$, is continuous linear with dense range 4 we have an injective adjoint map

$$
\iota^{\prime}: \mathcal{H}_{K} \rightarrow C^{-\infty}(M), \quad \iota^{\prime}(v)(\varphi):=\left\langle v, K_{\varphi}\right\rangle .
$$

In the following we accordingly identify $\mathcal{H}_{K}$ with the subspace $\iota^{\prime}\left(\mathcal{H}_{K}\right)$ of $C^{-\infty}(M)$. We then have the relation

$$
K_{\varphi}(\psi)=\left\langle K_{\varphi}, K_{\psi}\right\rangle=\langle\varphi, \psi\rangle_{K}=K(\bar{\varphi} \otimes \psi) \quad \text { for } \quad \varphi, \psi \in C_{c}^{\infty}(M) .
$$

The continuity of the linear map $\iota$ implies that the kernel $K$ is a continuous bilinear map, hence a smooth kernel on the linear locally convex manifold $C_{c}^{\infty}(M)$.

\footnotetext{
${ }^{4}$ To see that $\iota$ is continuous, it suffices to verify this claim on the subspace $\mathcal{D}_{C}(M)$ for a compact subset $C \subseteq M$. On this Fréchet space, the map $\mathcal{D}_{C}(M) \rightarrow \mathcal{D}_{C \times C}(M \times M), \varphi \mapsto \varphi \otimes \varphi$, is easily seen to be continuous. Therefore the continuity of $K$ implies that $\varphi \mapsto\langle\varphi, \varphi\rangle_{K}$ is bounded on some 0-neighborhood, and this means that $\left.\iota\right|_{\mathcal{D}_{C}(M)}$ is continuous.
} 
Therefore the results of Section 4 apply in particular to $K$ as a smooth kernel on $C_{c}^{\infty}(M)$.

Definition 7.2. The Lie derivative defines on $C_{c}^{\infty}(M)$ the structure of a $\mathcal{V}(M)$ module, and we consider on $C^{-\infty}(M)$ the adjoint representation:

$$
\left(\mathcal{L}_{X} D\right)(\varphi):=-D\left(\mathcal{L}_{X} \varphi\right) \quad \text { for } \quad X \in \mathcal{V}(M), D \in C^{-\infty}(M), \varphi \in C_{c}^{\infty}(M) .
$$

For a distribution $D \in C^{-\infty}(M \times M)$ and $X \in \mathcal{V}(M)$, we write

$$
\left(\mathcal{L}_{X}^{1} D\right)(\varphi \otimes \psi):=-D\left(\mathcal{L}_{X} \varphi \otimes \psi\right) \quad \text { and } \quad\left(\mathcal{L}_{X}^{2} D\right)(\varphi \otimes \psi):=-D\left(\varphi \otimes \mathcal{L}_{X} \psi\right)
$$

and we say that $X$ is D-symmetric if $\mathcal{L}_{X}^{1} D=\mathcal{L}_{X}^{2} D$ and D-skew-symmetric if $\mathcal{L}_{X}^{1} D=-\mathcal{L}_{X}^{2} D$.

Remark 7.3. Let $K$ be a positive definite distribution on $M$. If $X$ is $K$-symmetric (resp. $K$-skew-symmetric), then $\mathcal{L}_{X}$ defines a symmetric (resp. skew-symmetric) operator on $C_{c}^{\infty}(M)$ with respect to $\langle\cdot, \cdot\rangle_{K}$.

The next observation allows us to use Corollary 4.9 and to adapt the methods used in Section 5 .

Remark 7.4. Let $X \in \mathcal{V}(M)$ be locally integrable and $\varphi \in C_{c}^{\infty}(M)$. If $\operatorname{supp} \varphi \subseteq$ $M_{-t}$, then $\varphi \circ \Phi_{t}^{X}$ has compact support $\Phi_{-t}^{X}(\operatorname{supp} \varphi) \subseteq M_{t}$ and therefore can be seen as an element of $C_{c}^{\infty}(M)$.

Theorem 7.5 (Geometric Fröhlich Theorem for distributions). Let $M$ be a smooth manifold and let $K \in C^{-\infty}(M \times M)$ be a positive definite distribution. If $X \in \mathcal{V}(M)$ is a locally integrable $K$-symmetric vector field on $M$, then the Lie derivative $\mathcal{L}_{X}$ defines an essentially selfadjoint operator $\mathcal{H}_{K}^{0} \rightarrow \mathcal{H}_{K}$ whose closure $\mathcal{L}_{X}^{K}$ coincides with $\left.\mathcal{L}_{X}\right|_{\mathcal{D}_{X}}$, where

$$
\mathcal{D}_{X}:=\left\{D \in \mathcal{H}_{K}: \mathcal{L}_{X} D \in \mathcal{H}_{K}\right\} .
$$

Moreover, if the local flow $\Phi^{X}$ is defined on $[-\varepsilon, 0] \times \operatorname{supp}(\varphi)$ for some $\varphi \in C_{c}^{\infty}(M)$, then

$$
e^{t \mathcal{L}_{X}^{K}} K_{\varphi}=K_{\varphi \circ \Phi_{-t}^{X}} \quad \text { for } \quad 0 \leq t \leq \varepsilon .
$$

Proof. For every $\varphi \in C_{c}^{\infty}(M)$, there exists an $\varepsilon>0$ such that the flow $\Phi^{X}$ of $X$ is defined on the compact subset $[-\varepsilon, 0] \times \operatorname{supp}(\varphi)$ of $\mathbb{R} \times M$. Then the curve

$$
\gamma:[0, \varepsilon] \rightarrow C_{c}^{\infty}(M), \quad \gamma(t):=\varphi \circ \Phi_{-t}^{X}
$$

satisfies $\gamma^{\prime}(t)=-\mathcal{L}_{X} \varphi$ in the natural topological on $C_{c}^{\infty}(M)$. Therefore the assumptions of Corollary 4.9 are satisfied with $V=C_{c}^{\infty}(M)$ and $L=\mathcal{L}_{X}$. We conclude that $\left.\mathcal{L}_{X}\right|_{\mathcal{H}_{K}^{0}}$ is essentially selfadjoint with closure equal to $\mathcal{L}_{X}^{K}$ and that $e^{t \mathcal{L}_{X}^{K}} K_{\varphi}=K_{\varphi \circ \Phi_{-t}^{X}}$ for $0 \leq t \leq \varepsilon$.

\subsection{The Integrability Theorem for distributions.}

Definition 7.6. Let $\mathfrak{g}=\mathfrak{h}+\mathfrak{q}$ be a symmetric Lie algebra with involution $\tau$ and let $\beta: \mathfrak{g} \rightarrow \mathcal{V}(M)$ be a homomorphism of Lie algebras. A positive definite distribution $K \in C^{-\infty}(M \times M)$ is said to be $\beta$-compatible if

$$
\mathcal{L}_{\beta(x)}^{1} K=-\mathcal{L}_{\beta(\tau(x))}^{2} K \quad \text { for } \quad x \in \mathfrak{g} .
$$


In the following we assume that $K$ is a positive definite distribution on $M$ compatible with the smooth right action $(\beta, \sigma)$ of $(\mathfrak{g}, H)$ (cf. Definition 5.4). For $z \in \mathfrak{g}_{\mathbb{C}}$, we put

$$
\mathcal{L}_{\beta(z)}:=\mathcal{L}_{\beta(x)}+i \mathcal{L}_{\beta(y)}
$$

and we write $\mathcal{L}_{z}$ for the restriction of $\mathcal{L}_{\beta(z)}$ to its maximal domain

$$
\mathcal{D}_{z}=\left\{D \in \mathcal{H}_{K}: \mathcal{L}_{\beta(z)} D \in \mathcal{H}_{K}\right\} .
$$

As in Section 4, we define $\mathcal{D}^{1}:=\bigcap_{x \in \mathfrak{g}} \mathcal{D}_{x}$ and

$$
\mathcal{D}:=\left\{D \in \mathcal{H}_{K}:(\forall n \in \mathbb{N})\left(\forall x_{1}, \ldots, x_{n} \in \mathfrak{g}\right) \mathcal{L}_{\beta\left(x_{1}\right)} \cdots \mathcal{L}_{\beta\left(x_{n}\right)} D \in \mathcal{H}_{K}\right\}
$$

so that we obtain a Lie algebra representation $\alpha: \mathfrak{g}_{\mathbb{C}} \rightarrow \operatorname{End}(\mathcal{D})$ such that, for $x \in \mathfrak{g}^{c}:=\mathfrak{h}+i \mathfrak{q}$, the operator $\alpha(x)$ is skew-hermitian. From (4.4) and Remark 7.3 we deduce that

$$
\mathcal{L}_{x} K_{\varphi}=K_{\mathcal{L}_{\tau(x)} \varphi}
$$

hence $\mathcal{H}_{K}^{0} \subseteq \mathcal{D}$. In particular, $\mathcal{D}$ is dense in $\mathcal{H}_{K}$.

Lemma 7.7. The prescription $\pi^{H}(D)(\varphi):=D\left(\varphi \circ \sigma_{h}^{-1}\right)$ defines a smooth unitary representation $\left(\pi^{H}, \mathcal{H}_{K}\right)$ of $H$. For $h \in H$ and $\varphi \in C_{c}^{\infty}(M)$, we have

$$
\pi^{H}(h) K_{\varphi}:=K_{\varphi \circ \sigma_{h}}
$$

and $\mathcal{H}_{K}^{0}$ consists of smooth vectors. For $x \in \mathfrak{h}$, the infinitesimal generator $\overline{\mathrm{d} \pi^{H}}(x)$ of the unitary one-parameter group $\pi_{x}^{H}(t):=\pi^{H}(\exp t x)$ coincides with $\mathcal{L}_{x}$. Moreover $\mathcal{H}_{K}^{0}$ is a core for $\mathcal{L}_{x}$.

Proof. The $H$-invariance of $K$ implies that the hermitian form $\langle\cdot, \cdot\rangle_{K}$ on $C_{c}^{\infty}(M)$ is invariant under the action of $H$ on $C_{c}^{\infty}(M)$. This implies that the subspace $\mathcal{H}_{K} \subseteq$ $C^{-\infty}(M)$ is invariant under the natural $H$-action on $C^{-\infty}(M)$ given by $(h . D)(\varphi)=$ $D\left(\varphi \circ \sigma_{h}^{-1}\right)$ and that it restricts to a unitary representation (cf. [Ne00, Prop. II.4.9]). From (17.1) and the connectedness of $H$, we derive that

$$
h . K_{\varphi}=K_{\varphi \circ \sigma_{h}} \quad \text { for } \quad h \in H .
$$

The continuity of $\iota$ and the smoothness of the action of $H$ on $C_{c}^{\infty}(M)$ further imply that $\mathcal{H}_{K}^{0}$ consists of smooth vectors. The second part of the lemma follows from Lemma 4.4

Lemma 7.8. For each $D \in \mathcal{D}^{1}$, the complex linear map

$$
\omega_{D}: \mathfrak{g}_{\mathbb{C}} \rightarrow \mathcal{H}_{K}, \quad x \mapsto \mathcal{L}_{x} D
$$

is continuous.

Proof. Since $\mathfrak{g}_{\mathbb{C}}$ and $\mathcal{H}$ are Banach spaces, it suffices to show that the graph of $\omega_{D}$ is closed. This follows from the continuity of the linear functionals

$$
\mathfrak{g}_{\mathbb{C}} \rightarrow \mathbb{C}, \quad x \mapsto\left(\mathcal{L}_{x} D\right)(\varphi)=-D\left(\mathcal{L}_{x} \varphi\right), \quad \varphi \in C_{c}^{\infty}(M) .
$$

To show that the representation $\alpha: \mathfrak{g}^{c} \rightarrow \operatorname{End}(\mathcal{D})$ integrates to a continuous unitary representation of $G^{c}$ we will again use Theorem 5.9 .

Theorem 7.9. Let $K \in C^{-\infty}(M \times M)$ be a positive definite distribution compatible with the smooth right action $(\beta, \sigma)$ of the pair $(\mathfrak{g}, H)$ on $M$, where $\mathfrak{g}=\mathfrak{h} \oplus \mathfrak{q}$ is a symmetric Banach-Lie algebra and $H$ is a connected Lie group with Lie algebra $\mathfrak{h}$. Let $G^{c}$ be a simply connected Lie group with Lie algebra $\mathfrak{g}^{c}=\mathfrak{h}+i \mathfrak{q}$. Then there exists a unique smooth unitary representation $\left(\pi^{c}, \mathcal{H}_{K}\right)$ of $G^{c}$ such that 
(i): $\overline{\mathrm{d} \pi^{c}}(x)=\mathcal{L}_{x}$ for $x \in \mathfrak{h}$.

(ii): $\overline{\mathrm{d} \pi^{c}}(i y)=i \mathcal{L}_{y}$ for $y \in \mathfrak{q}$.

Proof. The proof is very similar to the one of Theorem5.12. The first step has to be slightly adapted. For $x \in \mathfrak{q}$, we consider the associated selfadjoint operator $\mathcal{L}_{x}$ on $\mathcal{H}_{K}$ (Theorem 4.6). Then we obtain by spectral calculus a hermitian one-parameter group $e^{t \mathcal{L}_{x}}$ of unbounded selfadjoint operators on $\mathcal{H}_{K}$ and we put $\mathcal{D}_{t}=\mathcal{D}\left(e^{t \mathcal{L}_{x}}\right)$. For $\varphi \in C_{c}^{\infty}(M)$ with $\operatorname{supp}(\varphi) \subseteq M_{s}$ we know from Theorem 7.5 that $K_{\varphi} \in \mathcal{D}_{s}$ with

$$
\gamma(t)=K_{\varphi \circ \Phi_{-t}^{\beta(x)}}=e^{t \mathcal{L}_{x}} K_{\varphi} \quad \text { for } \quad 0 \leq t \leq s .
$$

The curve $t \mapsto e^{t \mathcal{L}_{x}} K_{\varphi}$ in $\mathcal{H}_{K}$ extends to a holomorphic function $e^{z \mathcal{L}_{x}} K_{\varphi}$ defined on an open neighborhood of the strip $0 \leq \operatorname{Re} z \leq s$. Accordingly, for $y \in \mathfrak{g}$ and $\psi \in C_{c}^{\infty}(M)$, the function

extends to a holomorphic function

$$
t \mapsto\left\langle e^{t \mathcal{L}_{x}} K_{\varphi}, \mathcal{L}_{y} K_{\psi}\right\rangle
$$

$$
z \mapsto\left\langle e^{z \mathcal{L}_{x}} K_{\varphi}, \mathcal{L}_{y} K_{\psi}\right\rangle .
$$

For $x \in \mathfrak{q}, y \in \mathfrak{g}$ and $\varphi, \psi \in C_{c}^{\infty}(M)$ with $\operatorname{supp}(\varphi), \operatorname{supp}(\psi) \subseteq M_{s}$, we thus get:

$$
\begin{aligned}
\left\langle e^{t \mathcal{L}_{x}} K_{\varphi}, \mathcal{L}_{\tau(y)} K_{\psi}\right\rangle & =\left\langle K_{\varphi \circ \Phi_{-t}^{\beta(x)}}, \mathcal{L}_{\tau(y)} K_{\psi}\right\rangle=\overline{\left(\mathcal{L}_{\tau(y)} K_{\psi}\right)\left(\varphi \circ \Phi_{-t}^{\beta(x)}\right)} \\
& =\overline{\left(\mathcal{L}_{e^{t \operatorname{ad} x} \tau(y)} K_{\psi \circ \Phi^{\beta(x)}}\right)}(\varphi) \\
& =\left\langle K_{\varphi}, \mathcal{L}_{e^{t \text { ad } x} \tau(y)} e^{t \mathcal{L}_{x}} K_{\psi}\right\rangle \\
& =-\left\langle\mathcal{L}_{e^{-t \text { ad } x} y} K_{\varphi}, e^{t \mathcal{L}_{x}} K_{\psi}\right\rangle .
\end{aligned}
$$

By analytic extension (cf. [KL81, Lemma 2]), we now arrive with Lemma 7.8 at the relation

$$
\left\langle e^{z \mathcal{L}_{x}} K_{\varphi}, \mathcal{L}_{\tau(y)} K_{\psi}\right\rangle=-\left\langle\mathcal{L}_{e^{-z \text { ad } x} y} K_{\varphi}, e^{\bar{z} \mathcal{L}_{x}} K_{\psi}\right\rangle \quad \text { for } \quad 0 \leq \operatorname{Re} z \leq t
$$

and we get in particular

$$
\left\langle e^{i \mathcal{L}_{x}} K_{\varphi}, \mathcal{L}_{\tau(y)} K_{\psi}\right\rangle=-\left\langle\mathcal{L}_{e^{-i \text { ad } x} y} K_{\varphi}, e^{-i \mathcal{L}_{x}} K_{\psi}\right\rangle .
$$

This relation leads to $e^{i \mathcal{L}_{x}} K_{\varphi} \in \mathcal{D}_{y}$ with

$$
\mathcal{L}_{y} e^{i \mathcal{L}_{x}} K_{\varphi}=e^{i \mathcal{L}_{x}} \mathcal{L}_{e^{-i \operatorname{ad} x} y} K_{\varphi} \quad \text { for } \quad \varphi \in C_{c}^{\infty}(M), y \in \mathfrak{g} .
$$

Here we use that $M=\bigcup_{s>0} M_{s}$ implies that $\operatorname{supp}(\varphi) \subseteq M_{s}$ for some $s>0$. We thus obtain $e^{i \mathcal{L}_{x}} \mathcal{H}_{K}^{0} \subseteq \mathcal{D}^{1}$ with

resp.,

$$
\left.\mathcal{L}_{y} e^{i \mathcal{L}_{x}}\right|_{\mathcal{H}_{K}^{0}}=\left.e^{i \mathcal{L}_{x}} \mathcal{L}_{e^{-i \text { ad } x} y}\right|_{\mathcal{H}_{K}^{0}},
$$

$$
\left.e^{-i \mathcal{L}_{x}} \mathcal{L}_{y} e^{i \mathcal{L}_{x}}\right|_{\mathcal{H}_{K}^{0}}=\left.\mathcal{L}_{e^{-i \operatorname{ad} x} y}\right|_{\mathcal{H}_{K}^{0}} \quad \text { for } \quad x \in \mathfrak{q}, y \in \mathfrak{g}^{c}
$$

Now the rest of the proof is exactly as in Steps 2 and 3 of the proof of Theorem 5.12 .

Remark 7.10. Let $(\beta, \sigma)$ be a smooth right action of $(\mathfrak{g}, H)$ on $M$. Then $V:=$ $C_{c}^{\infty}(M)$ is a locally convex manifold on which the Lie derivatives $\mathcal{L}_{\beta(x)}$ defines a representation $\mathfrak{g} \rightarrow \mathcal{V}(V)$. Moreover, every positive definite distribution $K$ on $M \times M$ defines a smooth positive definite kernel $\widetilde{K}(\varphi, \psi):=K(\bar{\varphi} \otimes \psi)$ on $V$. One is therefore tempted to derive the results in Section 7 from Section 5 . However, this does not work directly because the linear vector fields on $V$ defined by the Lie 
derivatives are not locally integrable if the corresponding flow on $M$ is not global (cf. Proposition 2.9).

7.3. Reflection positive distributions and representations. In this subsection we connect the previously obtained integrability result to reflection positivity (cf. NO13, NO14,JO198, JO100]). We use the notation from [NO13, NO14].

Let $D \in C^{\infty}(M \times M, \mathbb{C})$ be a reflection positive distribution kernel which is reflection positive w.r.t. the involution $\theta: M \rightarrow M$ on the open subset $M_{+} \subseteq M$ (cf. Definition 7.13). Our main result is Theorem 7.14 which shows that, under the natural compatibility requirements for an action of a symmetric Lie group $(G, H, \tau)$ on $(M, \theta)$, the representation of the pair $\left(\mathfrak{g}^{c}, H\right)$ on the Hilbert space $\mathcal{H}_{D_{+}}$corresponding to the positive definite distribution $D(\cdot, \theta \cdot)$ on $M_{+}$integrates to a unitary representation of the corresponding simply connected group $G^{c}$ with Lie algebra $\mathfrak{g}^{c}$.

Definition 7.11. Let $\mathcal{E}$ be a Hilbert space and $\theta \in \mathrm{U}(\mathcal{E})$ be an involution. We call a closed subspace $\mathcal{E}_{+} \subseteq \mathcal{E} \theta$-positive if $\langle\theta v, v\rangle \geq 0$ for $v \in \mathcal{E}_{+}$. We then say that the triple $\left(\mathcal{E}, \mathcal{E}_{+}, \theta\right)$ is a reflection positive Hilbert space. In this case we write

$$
\mathcal{N}:=\left\{v \in \mathcal{E}_{+}:\langle\theta v, v\rangle=0\right\}=\left\{v \in \mathcal{E}_{+}:\left(\forall w \in \mathcal{E}_{+}\right)\langle\theta w, v\rangle=0\right\}=\mathcal{E}_{+} \cap \theta\left(\mathcal{E}_{+}\right)^{\perp},
$$

$q: \mathcal{E}_{+} \rightarrow \mathcal{E}_{+} / \mathcal{N}, v \mapsto \widehat{v}=q(v)$, for the quotient map and $\widehat{\mathcal{E}}$ for the Hilbert completion of $\mathcal{E}_{+} / \mathcal{N}$ with respect to the norm $\|\widehat{v}\|_{\widehat{\mathcal{E}}}:=\|\widehat{v}\|:=\sqrt{\langle\theta v, v\rangle}$. If $T: \mathcal{E}_{+} \rightarrow \mathcal{E}_{+}$is continuous and leaves $\mathcal{N}$ invariant, then we denote by $\widehat{T}: \widehat{\mathcal{E}} \rightarrow \widehat{\mathcal{E}}$ the corresponding operator on $\widehat{\mathcal{E}}$.

Let $(G, H, \tau)$ be a symmetric Lie group and $\mathfrak{g}=\mathfrak{h} \oplus \mathfrak{q}$ be the corresponding symmetric Lie algebra.

Definition 7.12. Let $(G, H, \tau)$ be a symmetric Lie group and $\left(\mathcal{E}, \mathcal{E}_{+}, \theta\right)$ be a reflection positive Hilbert space. A unitary representation $\pi: G \rightarrow \mathrm{U}(\mathcal{E})$ is said to be reflection positive on $\left(\mathcal{E}, \mathcal{E}_{+}, \theta\right)$ if the following three conditions hold:

(RP1) $\pi(\tau(g))=\theta \pi(g) \theta$ for every $g \in G$.

(RP2) $\pi(h) \mathcal{E}_{+}=\mathcal{E}_{+}$for every $h \in H$.

(RP3) There exists a subspace $\mathcal{D} \subseteq \mathcal{E}_{+} \cap \mathcal{E}^{\infty}$, dense in $\mathcal{E}_{+}$, such that $\mathrm{d} \pi(X) \mathcal{D} \subset \mathcal{D}$ for all $X \in \mathfrak{q}$.

If $\pi$ is a reflection positive representation on $\left(\mathcal{E}, \mathcal{E}_{+}, \theta\right)$, then it follows from [NO13, Lemma 2.4] that by defining $\widehat{\pi}_{0}(h)=\widehat{\pi(h)}$ (in the sense of Definition 7.11), we get a unitary representation $\left(\widehat{\pi}_{0}, \widehat{\mathcal{E}}\right)$ of $H$. However, we would like to have a unitary representation $\pi^{c}$ of $G^{c}$ on $\widehat{\mathcal{E}}$ "extending" $\widehat{\pi}_{0}$. In the following we give a geometric construction of reflection positive representations and we show, using the previous results, that they can be analytically continued to unitary representations of $G^{c}$.

Definition 7.13. Let $M$ be a smooth finite dimensional manifold and let $D \in$ $C^{-\infty}(M \times M)$ be a positive definite distribution. Suppose further that $\theta: M \rightarrow M$ is an involutive diffeomorphism and that $M_{+} \subseteq M$ is an open subset such that the distribution $D_{+}$on $M_{+} \times M_{+}$defined by

$$
D_{+}(\varphi):=D\left(\varphi \circ\left(\theta \times \operatorname{id}_{M}\right)\right), \quad \varphi \in C_{c}^{\infty}\left(M_{+} \times M_{+}\right) \subset C_{c}^{\infty}(M \times M)
$$

is positive definite. We then say that $D$ is reflection positive with respect to $\left(M, M_{+}, \theta\right)$. 
Let $D \in C^{-\infty}(M \times M)$ be a reflection positive distribution with respect to $\left(M, M_{+}, \theta\right)$ as in Definition 7.13 and let $\mathcal{E} \subseteq C^{-\infty}(M)$ be the corresponding reflection positive Hilbert space obtained by completing $C_{c}^{\infty}(M)$ with respect to the scalar product

$$
\langle\varphi, \psi\rangle_{D}:=D(\bar{\varphi} \otimes \psi)
$$

Then the closed subspace $\mathcal{E}_{+}$generated by $C_{c}^{\infty}\left(M_{+}\right)$is $\theta$-positive with respect to $\theta \varphi:=\varphi \circ \theta$ and $\widehat{\mathcal{E}} \cong \mathcal{H}_{D_{+}} \subseteq C_{c}^{-\infty}\left(M_{+}\right)$(cf. NO13).

Let $(G, H, \tau)$ be a symmetric Lie group acting on $M$ such that $\theta(g . m)=\tau(g) \cdot \theta(m)$ and $H . M_{+}=M_{+}$. We assume that $D$ is invariant under $G$ and $\tau$. Then we have a natural unitary representation $\left(\pi_{\mathcal{E}}, \mathcal{E}\right)$ of $G$ given by

$$
\left(\pi_{\mathcal{E}}(g) E\right)(\varphi):=E\left(\varphi \circ \sigma_{g}\right) \quad \text { for } \quad E \in \mathcal{E} \subseteq C^{-\infty}(M), \varphi \in C_{c}^{\infty}(M),
$$

where $\sigma_{g}(m)=g . m$. As $M_{+}$, and therefore $\mathcal{E}_{+}$, is $H$-invariant, this representation is reflection positive.

From the invariance condition

$$
\mathcal{L}_{\beta(x)}^{1} D=-\mathcal{L}_{\beta(x)}^{2} D \quad \text { for } \quad x \in \mathfrak{g},
$$

we derive

$$
\mathcal{L}_{\beta(x)}^{1} D_{+}=-\mathcal{L}_{\beta(\tau(x))}^{2} D_{+} \quad \text { for } \quad x \in \mathfrak{g} .
$$

This implies that the assumptions of Theorem 7.9 are satisfied, so that we obtain the following theorem. It provides a very direct integrability theorem for representations of $(\mathfrak{g}, H)$ obtained from reflection positive distributions.

Theorem 7.14. Let $M$ be a smooth finite dimensional manifold and let $D \in$ $C^{-\infty}(M \times M)$ be a positive definite distribution which is reflection positive w.r.t. $\left(M, M_{+}, \theta\right)$. Let $(G, H, \tau)$ be a symmetric Lie group acting on $M$ such that $\theta(g . m)=$ $\tau(g) \cdot \theta(m)$ and $H \cdot M_{+}=M_{+}$. We assume that $D$ is invariant under $G$ and $\tau$. Let $G^{c}$ be a simply connected Lie group with Lie algebra $\mathfrak{g}^{c}=\mathfrak{h}+i \mathfrak{q}$ and define $\mathcal{L}_{x}$, $x \in \mathfrak{g}$, on its maximal domain in the Hilbert subspace $\mathcal{H}_{D_{+}} \subseteq C^{-\infty}\left(M_{+}\right)$. Then there exists a unique smooth unitary representation $\left(\pi^{c}, \mathcal{H}_{D_{+}}\right)$of $G^{c}$ such that

(i) $\overline{\mathrm{d} \pi^{c}}(x)=\mathcal{L}_{x}$ for $x \in \mathfrak{h}$.

(ii) $\overline{\mathrm{d} \pi^{c}}(i y)=i \mathcal{L}_{y}$ for $y \in \mathfrak{q}$.

Example 7.15. Reflection positive representations for $\operatorname{Mot}\left(\mathbb{R}^{d}\right)$ lead in particular to reflection positive representations of the compact symmetric Lie group $(G, H, \tau)=\left(\mathrm{O}_{d}(\mathbb{R}), \mathrm{O}_{d-1}(\mathbb{R}), \tau\right)$ which then leads to unitary representations of the $c$-dual group $G^{c}=\mathrm{SO}_{1, d-1}(\mathbb{R})_{0}=L_{+}^{\uparrow}$. In particular, there are non-trivial reflection positive representations for compact groups. Here the case $d=2$ and the free spin zero fields of mass $m>0$ are of particular interest (cf. [NO13]).

Example 7.16. We now consider $M=\mathbb{R}^{d}, M_{+}=\mathbb{R}_{+}^{d}, \tau\left(x_{0}, \mathbf{x}\right)=\left(-x_{0}, \mathbf{x}\right)$, and the euclidean motion group $G=\mathbb{R}^{d} \rtimes \mathrm{O}_{d}(\mathbb{R})$. Then the $G$-invariance of a distribution $D^{\sharp}$ on $M \times M$ means that it is determined by an $\mathrm{O}_{d}(\mathbb{R})$-invariant distribution $D \in C^{-\infty}(M)$ by

$$
D^{\sharp}(\varphi \otimes \psi):=D(\check{\varphi} * \psi) .
$$

For any reflection positive rotation invariant distribution $D \in C^{-\infty}\left(\mathbb{R}^{d}\right)$, we thus obtain a reflection positive representation $\left(\pi_{\mathcal{E}}, \mathcal{E}\right)$ of $G$ and a representation of the group $G^{c}=\mathbb{R}^{d} \rtimes \operatorname{Spin}_{1, d-1}(\mathbb{R})$ on $\widehat{\mathcal{E}} \cong \mathcal{H}_{D_{+}}$. 
The inclusion $\mathrm{SO}_{d-1}(\mathbb{R}) \rightarrow \mathrm{O}_{1, d-1}(\mathbb{R}), g \mapsto \mathrm{id}_{\mathbb{R}} \times g$, induces a surjective homomorphism $\pi_{1}\left(\mathrm{SO}_{d-1}(\mathbb{R})\right) \rightarrow \pi_{1}\left(\mathrm{O}_{1, d-1}(\mathbb{R})\right)$, and, since $\pi^{c}$ is compatible with the unitary representation $\widehat{\pi}_{H}$ of $H$ on $\widehat{\mathcal{E}}$, it follows that $\pi^{c}$ factors through a representation of the connected Poincaré group $\mathbb{R}^{d} \rtimes \mathrm{SO}_{1, d-1}(\mathbb{R})$.

\section{Appendix A. Positive definite KeRnels And Functions}

In this appendix we collect some definitions and results concerning positive definite functions and kernels.

Definition A.1. Let $X$ be a set and $\mathcal{F}$ be a complex Hilbert space.

(a) A function $K: X \times X \rightarrow B(\mathcal{F})$ is called a $B(\mathcal{F})$-valued kernel. A $B(\mathcal{F})$ valued kernel $K$ on $X$ is said to be positive definite if, for every finite sequence $\left(x_{1}, v_{1}\right), \ldots,\left(x_{n}, v_{n}\right)$ in $X \times \mathcal{F}$,

$$
\sum_{j, k=1}^{n}\left\langle K\left(x_{j}, x_{k}\right) v_{k}, v_{j}\right\rangle \geq 0 .
$$

(b) If $(S, *)$ is an involutive semigroup, then a function $\varphi: S \rightarrow B(\mathcal{F})$ is called positive definite if the kernel $K_{\varphi}(s, t):=\varphi\left(s t^{*}\right)$ is positive definite.

Positive definite kernels can be characterized as those for which there exists a Hilbert space $\mathcal{H}$ and a function $\gamma: X \rightarrow B(\mathcal{H}, \mathcal{F})$ such that

$$
K(x, y)=\gamma(x) \gamma(y)^{*} \quad \text { for } \quad x, y \in X
$$

(cf. [Ne00, Thm. I.1.4]). Here one may assume that the vectors $\gamma(x)^{*} v, x \in X, v \in$ $\mathcal{F}$, span a dense subspace of $\mathcal{H}$. If this is the case, then the pair $(\gamma, \mathcal{H})$ is called a realization of $K$. The map $\Phi: \mathcal{H} \rightarrow \mathcal{F}^{X}, \Phi(v)(x):=\gamma(x) v$, then realizes $\mathcal{H}$ as a Hilbert subspace of $\mathcal{F}^{X}$ with continuous point evaluations ev ev $_{x} \mathcal{H} \rightarrow \mathcal{F}, f \mapsto f(x)$. Then $\Phi(\mathcal{H})$ is the unique Hilbert space in $\mathcal{F}^{X}$ with continuous point evaluations $\mathrm{ev}_{x}$, for which $K(x, y)=\mathrm{ev}_{x} \mathrm{ev}_{y}^{*}$ for $x, y \in X$. We write $\mathcal{H}_{K} \subseteq \mathcal{F}^{X}$ for this subspace and call it the reproducing kernel Hilbert space with kernel $K$. The dense subspace spanned by the elements of the form $K_{m}^{*} v, v \in \mathcal{F}, m \in M$, is denoted $\mathcal{H}_{K}^{0}$.

For $\mathcal{F}=\mathbb{C}$, we also write $K_{m}$ for the function (which corresponds to $K_{m}^{*} 1$ ) which represents the evaluation in $m$ in the sense that $f(m)=\left\langle f, K_{m}\right\rangle$ for $f \in \mathcal{H}_{K}$.

Example A.2 (Vector-valued GNS construction). (cf. [Ne00, Sect. 3.1]) Let $(\pi, \mathcal{H})$ be a representation of the unital involutive semigroup $(S, *), \mathcal{F} \subseteq \mathcal{H}$ be a closed subspace for which $\pi(S) \mathcal{F}$ is total in $\mathcal{H}$ and $P: \mathcal{H} \rightarrow \mathcal{F}$ denote the orthogonal projection. Then $\varphi(s):=P \pi(s) P^{*}$ is a $B(\mathcal{F})$-valued positive definite function on $S$ with $\varphi(\mathbf{1})=\mathbf{1}_{\mathcal{F}}$ because $\gamma(s):=P \pi(s) \in B(\mathcal{H}, \mathcal{F})$ satisfies

$$
\gamma(s) \gamma(t)^{*}=P \pi\left(s t^{*}\right) P^{*}=\varphi\left(s t^{*}\right) .
$$

The map

$$
\Phi: \mathcal{H} \rightarrow \mathcal{F}^{S}, \quad \Phi(v)(s)=\gamma(s) v=P \pi(s) v
$$

is an $S$-equivariant realization of $\mathcal{H}$ as the reproducing kernel space $\mathcal{H}_{\varphi} \subseteq \mathcal{F}^{S}$, on which $S$ acts by right translation, i.e., $\left(\pi_{\varphi}(s) f\right)(t)=f(t s)$.

Conversely, let $S$ be a unital involutive semigroup and $\varphi: S \rightarrow B(\mathcal{F})$ be a positive definite function with $\varphi(\mathbf{1})=\mathbf{1}_{\mathcal{F}}$. Write $\mathcal{H}_{\varphi} \subseteq \mathcal{F}^{S}$ for the corresponding reproducing kernel space and $\mathcal{H}_{\varphi}^{0}$ for the dense subspace spanned by $\mathrm{ev}_{s}^{*} v, s \in S, v \in$ $\mathcal{F}$. Then $\left(\pi_{\varphi}(s) f\right)(t):=f(t s)$ defines a $*$-representation of $S$ on $\mathcal{H}_{\varphi}^{0}$. We say that $\varphi$ is exponentially bounded if all operators $\pi_{\varphi}(s)$ are bounded, so that we actually 
obtain a representation of $S$ by bounded operators on $\mathcal{H}_{\varphi}$. As $\mathbf{1}_{\mathcal{F}}=\varphi(\mathbf{1})=\mathrm{ev}_{\mathbf{1}} \mathrm{ev}_{\mathbf{1}}^{*}$, the map ev $\mathrm{v}_{\mathbf{1}}^{*}: \mathcal{F} \rightarrow \mathcal{H}$ is an isometric inclusion, so that we may identify $\mathcal{F}$ with a subspace of $\mathcal{H}$. Then $\mathrm{ev}_{\mathbf{1}}: \mathcal{H} \rightarrow \mathcal{F}$ corresponds to the orthogonal projection onto $\mathcal{F}$ and $\mathrm{ev}_{\mathbf{1}} \circ \pi_{\varphi}(s)=\mathrm{ev}_{s}$ leads to

$$
\varphi(s)=\mathrm{ev}_{s} \mathrm{ev}_{\mathbf{1}}^{*}=\mathrm{ev}_{\mathbf{1}} \pi_{\varphi}(s) \mathrm{ev}_{\mathbf{1}}^{*} .
$$

If $S=G$ is a group with $s^{*}=s^{-1}$, then $\varphi$ is always exponentially bounded and the representation $\left(\pi_{\varphi}, \mathcal{H}_{\varphi}\right)$ is unitary.

\section{REFERENCES}

[B95] E. Borel, Sur quelques points de la théorie des fonctions (French), Ann. Sci. École Norm. Sup. (3) 12 (1895), 9-55. MR 1508908

[Fro80] J. Fröhlich, Unbounded, symmetric semigroups on a separable Hilbert space are essentially selfadjoint, Adv. in Appl. Math. 1 (1980), no. 3, 237-256, DOI 10.1016/01968858(80)90012-3. MR603131 (82c:47044)

[FOS83] J. Fröhlich, K. Osterwalder, and E. Seiler, On virtual representations of symmetric spaces and their analytic continuation, Ann. of Math. (2) 118 (1983), no. 3, 461-489, DOI 10.2307/2006979. MR727701 (85j:22024)

[Jo86] P. E. T. Jorgensen, Analytic continuation of local representations of Lie groups, Pacific J. Math. 125 (1986), no. 2, 397-408. MR863534 (88m:22030)

[Jo87] P. E. T. Jorgensen, Analytic continuation of local representations of symmetric spaces, J. Funct. Anal. 70 (1987), no. 2, 304-322, DOI 10.1016/0022-1236(87)90115-7. MR874059 (88d:22021)

[JO198] P. E. T. Jorgensen and G. Ólafsson, Unitary representations of Lie groups with reflection symmetry, J. Funct. Anal. 158 (1998), no. 1, 26-88, DOI 10.1006/jfan.1998.3285. MR:1641554 (99m:22013)

[JO100] P. E. T. Jorgensen and G. Ólafsson, Unitary representations and Osterwalder-Schrader duality, The mathematical legacy of Harish-Chandra (Baltimore, MD, 1998), Proc. Sympos. Pure Math., vol. 68, Amer. Math. Soc., Providence, RI, 2000, pp. 333-401, DOI 10.1090/pspum/068/1767902. MR.1767902 (2001f:22036)

[KL81] A. Klein and L. J. Landau, Construction of a unique selfadjoint generator for a symmetric local semigroup, J. Funct. Anal. 44 (1981), no. 2, 121-137, DOI 10.1016/00221236(81)90007-0. MR642913 (83b:47051)

[KL82] A. Klein and L. J. Landau, From the Euclidean group to the Poincaré group via Osterwalder-Schrader positivity, Comm. Math. Phys. 87 (1982/83), no. 4, 469-484. MR691039 (84g:22043)

[LM75] M. Lüscher and G. Mack, Global conformal invariance in quantum field theory, Comm. Math. Phys. 41 (1975), 203-234. MR0371282 (51 \#7503)

[Mer11] S. Merigon, Integrating representations of Banach-Lie algebras, J. Funct. Anal. 260 (2011), no. 5, 1463-1475, DOI 10.1016/j.jfa.2010.10.011. MR2749434(2012a:22036)

[MN12] S. Merigon and K.-H. Neeb, Analytic extension techniques for unitary representations of Banach-Lie groups, Int. Math. Res. Not. IMRN 18 (2012), 4260-4300, DOI 10.1093/imrn/rnr174. MR2975382

[MN14] S. Mergon and K.-H. Neeb, Semibounded unitary representations of mapping groups with values in infinite dimensional hermitian groups, in preparation

[Ne00] K.-H. Neeb, Holomorphy and convexity in Lie theory, de Gruyter Expositions in Mathematics, vol. 28, Walter de Gruyter \& Co., Berlin, 2000. MR.1740617 (2001j:32020)

[Ne06] K.-H. Neeb, Towards a Lie theory of locally convex groups, Jpn. J. Math. 1 (2006), no. 2, 291-468, DOI 10.1007/s11537-006-0606-y. MR2261066 (2007k:22020)

[Ne10] K.-H. Neeb, On differentiable vectors for representations of infinite dimensional Lie groups, J. Funct. Anal. 259 (2010), no. 11, 2814-2855, DOI 10.1016/j.jfa.2010.07.020. MR2719276 (2012b:22031)

[Ne13] K.-H. Neeb, Unitary representations of unitary groups, "Lie theory workshops", Eds. G. Mason, I. Penkov, J. Wolf, "Developments in Math." Vol. 37, Springer, 2014, 197-243.

[NO14] K.-H. Neeb and G. Ólafsson, Reflection positivity and conformal symmetry, J. Funct. Anal. 266 (2014), no. 4, 2174-2224, DOI 10.1016/j.jfa.2013.10.030. MR 3150157 
[NO13] K.-H. Neeb and G. Ólafsson, Reflection positive one-parameter groups and dilations, Complex Analysis and Operator Theory, to appear.

[Nel59] E. Nelson, Analytic vectors, Ann. of Math. (2) 70 (1959), 572-615. MR0107176 (21 \#5901)

[Ol84] G. I. Ol'shanskiŭ, Infinite-dimensional classical groups of finite R-rank: description of representations and asymptotic theory (Russian), Funktsional. Anal. i Prilozhen. 18 (1984), no. 1, 28-42. MR.739087 (86a:22037)

[O190] G. I. Ol'shanskiü, Unitary representations of infinite-dimensional pairs $(G, K)$ and the formalism of $R$. Howe, Representation of Lie groups and related topics, Adv. Stud. Contemp. Math., vol. 7, Gordon and Breach, New York, 1990, pp. 269-463. MR.1104279 (92c:22043)

[OS73] K. Osterwalder and R. Schrader, Axioms for Euclidean Green's functions, Comm. Math. Phys. 31 (1973), 83-112. MR0329492 (48 \#7834)

[Pr92] H. Prado, Reflection positivity for unitary representations of Lie groups, Proc. Amer. Math. Soc. 114 (1992), no. 3, 723-731, DOI 10.2307/2159396. MR1072089 (92f:22019)

[Sch86] R. Schrader, Reflection positivity for the complementary series of SL $(2 n, \mathbf{C})$, Publ. Res. Inst. Math. Sci. 22 (1986), no. 1, 119-141, DOI 10.2977/prims/1195178376. MR.834352 (87h:81111)

[Sh84] D. S. Shucker, Extensions and generalizations of a theorem of Widder and of the theory of symmetric local semigroups, J. Funct. Anal. 58 (1984), no. 3, 291-309, DOI 10.1016/0022-1236(84)90044-2. MR759101 (86b:47077)

[Si72] J. Simon, On the integrability of representations of infinite dimensional real Lie algebras, Comm. Math. Phys. 28 (1972), 39-46. MR0308333 (46 \#7447)

Department of Mathematics, Friedrich-Alexander University, Erlangen-Nuremberg, Cauerstrasse 11, 91058 Erlangen, Germany

E-mail address: merigon@math.fau.de

Department of Mathematics, Friedrich-Alexander University, Erlangen-Nuremberg, Cauerstrasse 11, 91058 Erlangen, Germany

E-mail address: neeb@math.fau.de

Department of Mathematics, Louisiana State University, Baton Rouge, Louisiana 70803

E-mail address: olafsson@math.lsu.edu 